AUTHOR QUERY FORM

\begin{tabular}{|l|l|l|}
\hline \multicolumn{1}{|c|}{} & Journal: CAR & Please e-mail or fax your responses and any corrections to: \\
$\begin{array}{l}\text { E } \\
\text { ELSEVIER }\end{array}$ & Article Number: 5852 & F-mail: corrections.essd@elsevier.sps.co.in \\
\hline
\end{tabular}

Dear Author,

Please check your proof carefully and mark all corrections at the appropriate place in the proof (e.g., by using on-screen annotation in the PDF file) or compile them in a separate list. To ensure fast publication of your paper please return your corrections within 48 hours.

For correction or revision of any artwork, please consult http://www.elsevier.com/artworkinstructions.

Any queries or remarks that have arisen during the processing of your manuscript are listed below and highlighted by flags in the proof. Click on the ' $Q$ ' link to go to the location in the proof.

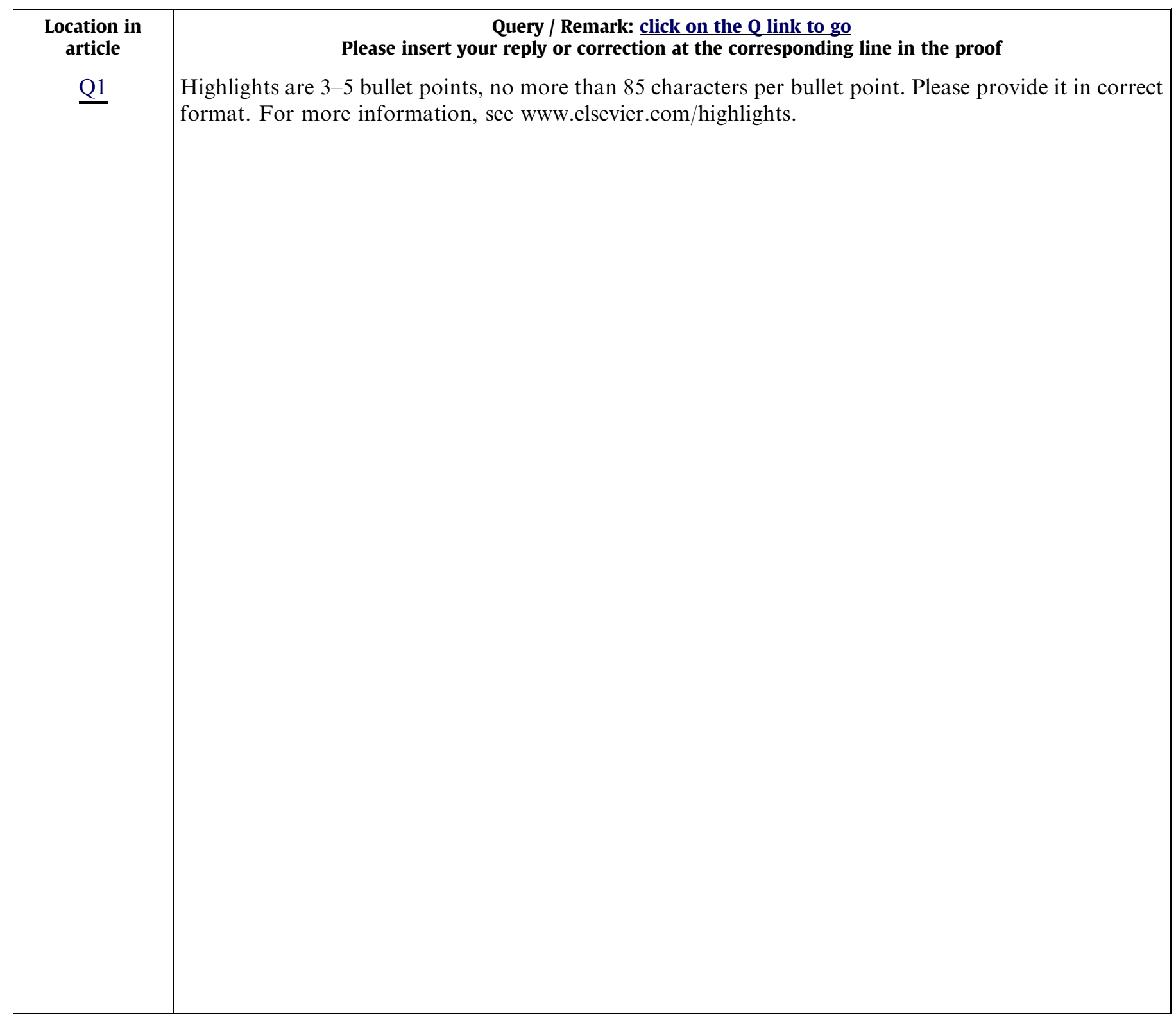

Thank you for your assistance. 


\section{Graphical abstract}

Ritter-type reaction of C-(1-bromo-1-deoxy-D-glycopyranosyl)formamides and its application for the synthesis of oligopeptides incorporating anomeric $\alpha$-amino acids

Katalin Czifrák, Viktor Gyóllai, Katalin E. Kövér, László Somsák ${ }^{*}$

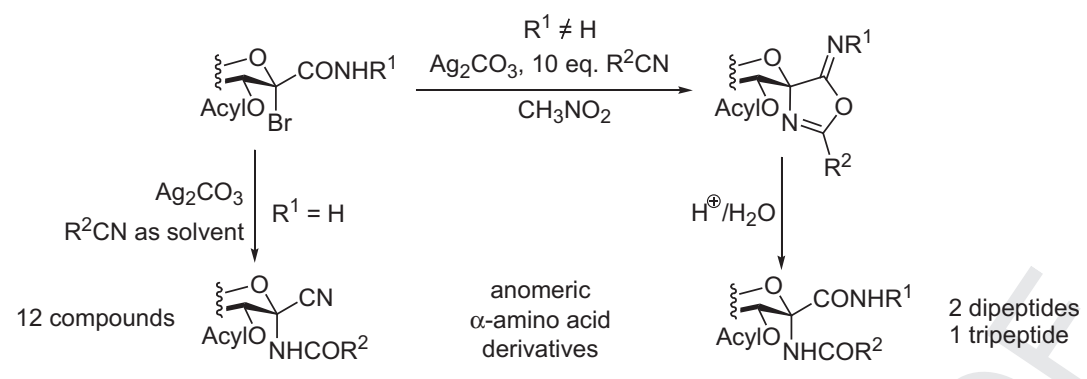




\title{
Ritter-type reaction of C-(1-bromo-1-deoxy-D-glycopyranosyl)formamides and its application for the synthesis of oligopeptides incorporating anomeric $\alpha$-amino acids
}

\author{
Katalin Czifrák, Viktor Gyóllai, Katalin E. Kövér, László Somsák* \\ Department of Organic Chemistry, University of Debrecen, POB 20, H-4010 Debrecen, Hungary
}

\section{A R T I C L E I N F O}

\section{Article history:}

Received 30 May 2011

Received in revised form 29 June 2011

Accepted 1 July 2011

Available online $\mathrm{xxxx}$

\section{Keywords:}

Ritter-reaction

2-Bromo-2-deoxy-hept(hex)-2-

ulopyranosonamide

2-Amino-2-deoxy-hept(hex)-2-

ulopyranosononitrile

Anomeric $\alpha$-amino acid

Oligopeptide

\begin{abstract}
A B S T R A C T
O-Peracetylated or -perbenzoylated C-(1-bromo-1-deoxy-D-glycopyranosyl)formamides of D-gluco, D-galacto, and D-arabino configuration were reacted with $\mathrm{Ag}(\mathrm{I})$-salts or $\mathrm{HgO}$ in nitrile solvents to give $\mathrm{N}$-acyl-1-cyano-D-glycopyranosylamines with an axial C-N bond at the anomeric centre. In the presence of $\mathrm{HgBr}_{2}, \mathrm{Hg}(\mathrm{CN})_{2}$, or $\mathrm{InCl}_{3}$ the anomer of the above glycosylamine with an equatorial $\mathrm{C}-\mathrm{N}$ bond was also isolated or detected. In $\mathrm{CH}_{3} \mathrm{NO}_{2}$ solutions as few as 5-10 equiv of the nitrile were sufficient to get acceptable yields for the products. Under similar conditions $\mathrm{N}$-substituted $C$-(2,3,4,6-tetra-O-acetyl-1-bromo-1deoxy- $\beta$-D-galactopyranosyl)formamides gave anomeric spiro-oxazoline derivatives which, upon mild acidic hydrolysis, opened up to di- and tripeptides of anomeric $\alpha$-amino acids.
\end{abstract}

\section{Introduction}

Ritter-type reactions involve combination of a carbocation (carbenium ion) with a nitrile (generally applied in high excess or as the solvent) to give a nitrilium ion which, after ensuing transformations by nucleophiles, may furnish carboxamides as well as heterocycles. ${ }^{1,2}$ Glycosyl-nitrilium ions (II formed from glycosylium ions I and nitriles, Scheme 1) are known to play important roles in directing the stereoselectivity of glycosylation reactions towards the formation of equatorial glycosides. ${ }^{3}$ On the other hand, attack of various external or internal nucleophiles onto glycosyl-nitrilium ions lead to several types of products rendering these transformations highly valuable in the carbohydrate field as well. Protecting groups in the sugar moieties can have a bearing on the configuration of glycosyl-nitrilium ions which tend to be axial (II) in the presence of non-participating protective groups demonstrated by NMR and computational methods ${ }^{4}$ as well as by the structure of the endproducts (vide infra). However, participating protection of the substituent in position 2 may force the formation of equatorial glycosyl-nitrilium ions (III). In these cases high equatorial selectivity can be observed in the products but, interestingly, exclusive axial selectivities were also reported in some transformations.

\footnotetext{
* Corresponding author. Tel.: +36 52512 900/22348; fax: +36 52512744 .

E-mail address: somsak@tigris.unideb.hu (L. Somsák).
}

Thus, in the presence of non-participating 2-substituents, axial $\mathrm{N}$-glycopyranosylamides were obtained from type II intermediates on the action of water, ${ }^{5}$ and $\mathrm{N}, \mathrm{N}$-bis-acylamide products were formed with several aromatic carboxylic acids ${ }^{6-8}$ and amino acids $^{9-11}$ as external nucleophiles. Carboxylic acids were also used as internal nucleophiles for the synthesis of several anomeric $\beta$-amino acid and peptide derivatives. ${ }^{12-15}$ Further internal O-nucleophiles, such as a $2-\mathrm{O}$-Zn salt obtained from a 1,2-epoxide $^{16}$ and a 2 -O-benzyl group ${ }^{17}$ gave 1,2 -annelated oxazolines, while the $\mathrm{CH}_{2} \mathrm{OH}$ appendage of heptulopyranose ${ }^{18}$ or fructopyranose ${ }^{19}$ derivatives furnished spiro-oxazolines, each with an axial $\mathrm{C}-\mathrm{N}$ bond at the anomeric carbon. 2- $\mathrm{N}$-Substituents were also observed to attack the axial glycosyl-nitrilium ion and furnished 1,2 -annelated imidazolines. ${ }^{20,21}$

With participating substituents next to the anomeric carbon, the outcome of the reactions is less predictable. With amino acids as well as aromatic carboxylic acids as external nucleophiles 2-deoxy-2-phthalimido ${ }^{22}$ and 2-deoxy-2-tetrachlorophthalimido ${ }^{11}$ D-glucopyranosyl derivatives gave equatorial $\mathrm{N}, \mathrm{N}$-bis-acylamide type products. Starting from $O$-perbenzoylated D-glucose, the equatorial amide was formed in low yield accompanied by several by-products in the presence of water as the nucleophile. ${ }^{23}$ Under similar conditions $O$-peracetylated D-glucose, D-galactose, and D-mannose each gave mixtures of axial and equatorial amides with a large excess of the latter. To explain this for the D-mannose case, equilibration of the amides was invoked. ${ }^{24}$ The internal 


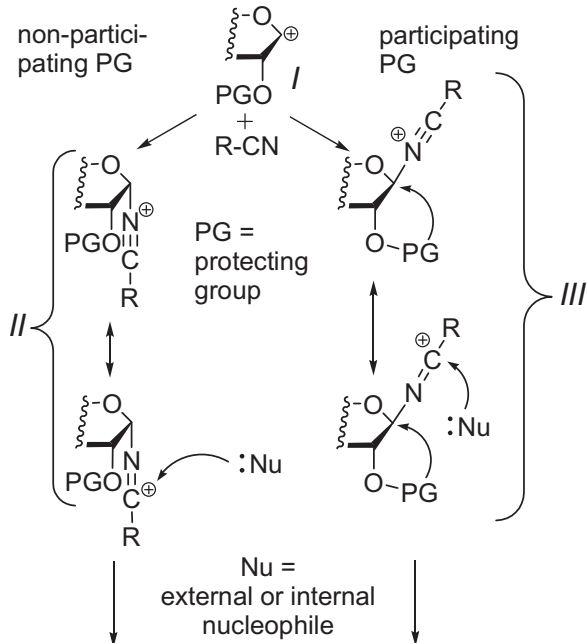

product with axial C-N bond (e.g. $\alpha$-D-gluco type) product with equatorial $\mathrm{C}-\mathrm{N}$ bond (e.g. $\beta$-D-gluco type) may be preponderant
Scheme 1.

nucleophile $\mathrm{CH}_{2} \mathrm{OH}$ group of a 3-O-benzoyl D-fructopyranose derivative gave only one spiro-oxazoline with an axial $\mathrm{C}-\mathrm{N}$ bond. ${ }^{19}$

Unprotected sugars were reported to give 1,2-cis configured $\mathrm{N}$-glycosylamides in liquid $\mathrm{HF}$ with both furanoid and pyranoid rings depending on the sugar configuration. ${ }^{25} \mathrm{D}$-Glucose was converted to $N$ - $\beta$-D-glucopyranosylamides with several nitriles in the presence of TMSOTf- $\mathrm{AgClO}_{4}$ under mechanochemical conditions. ${ }^{26}$

Some years ago we reported on the facile transformation of C-(1-bromo-1-deoxy-D-glycopyranosyl)formamides into $\mathrm{N}$-acyl-1-
cyano-D-glycopyranosylamines in the presence of $\mathrm{Ag}_{2} \mathrm{CO}_{3}$ in nitriles as solvents. ${ }^{27}$ In this paper, a detailed investigation of this reaction and its extension to the synthesis of some oligopeptide derivatives with an anomeric $\alpha$-amino acid moiety are presented.

\section{Results and discussion}

A Ritter-type reaction of C-(2,3,4,6-tetra-O-acetyl-1-bromo-1deoxy- $\beta$-D-galactopyranosyl)formamide ${ }^{28}(\mathbf{1}$, Table 1 , entry 1$)$ was first observed during an attempted exchange of the bromine to fluorine by $\mathrm{AgF}$ in dry $\mathrm{CH}_{3} \mathrm{CN}$. Under such conditions, widely applied for the synthesis of glycosyl fluoride derivatives (see Refs. 29-31 and references cited therein), an unexpected product, actually compound $\mathbf{2}$ was isolated in $70 \%$ yield instead of the expected $C$-(2,3,4,6-tetra- $O$-acetyl-1-deoxy-1-fluoro- $\alpha$-D-galactopyranosyl)formamide. In the presence of $\mathrm{Ag}_{2} \mathrm{CO}_{3} \mathbf{1}$ was transformed to 2 in a very clean reaction (entry 2 ), while AgOTf gave the same result under significantly shorter reaction time (entry 6 ). The new product crystallized out during removal of the solvent after the usual workup, did not exhibit characteristic fluoride couplings, had one exchangeable proton, and five methyl resonances in its ${ }^{1} \mathrm{H}$ NMR spectrum, and showed a CN signal in the ${ }^{13} \mathrm{C}$ NMR spectrum. Vicinal proton-proton couplings indicated that the sugar ring adopted a ${ }^{4} C_{1}$ conformation. The $\mathrm{CN}$ resonance appeared as a pseudo triplet in the proton coupled carbon spectrum with $\sim 3 \mathrm{~Hz}$ splittings due to couplings with $\mathrm{H}-2$ and the $\mathrm{NH}$ protons. This allowed us to deduce the equatorial orientation for the $\mathrm{CN}$ group based on the ${ }^{3} J_{\mathrm{H}-2, \mathrm{CN}}$ coupling in the ${ }^{4} C_{1}$ conformation. ${ }^{32-34}$

Some other promoters ${ }^{3}$ were also tried to perform this transformation. $\mathrm{HgO}$ (entry 7) gave similar results to those obtained with silver salts, but in the presence of $\mathrm{HgBr}_{2}, \mathrm{HgO}-\mathrm{HgBr}_{2}, \mathrm{Hg}(\mathrm{CN})_{2}$. and $\mathrm{InCl}_{3}$ (entries $8,12,13$, and 17 , respectively) a second product identified as $\mathbf{3}$, the anomer of $\mathbf{2}$, was also detected and isolated from the mixtures. Appearance of $\mathbf{4}^{35}$ in the reaction conducted

Table 1

Reaction of $C$-(2,3,4,6-tetra-O-acetyl-1-bromo-1-deoxy- $\beta$-D-galactopyranosyl)formamide (1) with $\mathrm{CH}_{3} \mathrm{CN}$ under various conditions

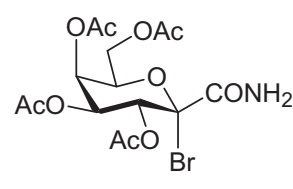

1

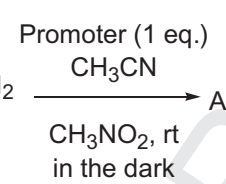

in the dark

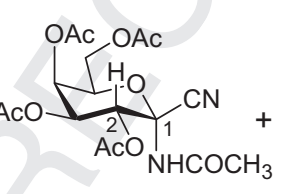

2

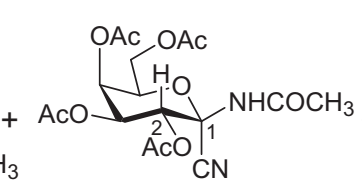

3

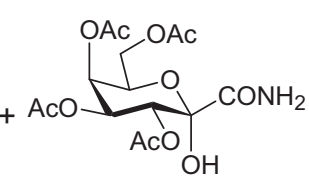

4

\begin{tabular}{|c|c|c|c|c|c|c|}
\hline \multirow[t]{2}{*}{ Entry } & \multirow[t]{2}{*}{ Promoter } & \multirow[t]{2}{*}{$\mathrm{CH}_{3} \mathrm{CN}$ (equiv) } & \multirow[t]{2}{*}{ Reaction time } & \multicolumn{3}{|c|}{ Product ratio (\%) by ${ }^{1} \mathrm{H}$ NMR } \\
\hline & & & & 2 & 3 & 4 \\
\hline 1 & $\mathrm{AgF}$ & As solvent & $3 d$ & $70^{\mathrm{a}, \mathrm{b}}$ & - & - \\
\hline 2 & $\mathrm{Ag}_{2} \mathrm{CO}_{3}$ & As solvent & $3 d$ & 100 & - & - \\
\hline 3 & & 10 & $5 \mathrm{~d}$ & 77 & - & 23 \\
\hline 4 & & 5 & $7 d$ & 68 & - & 32 \\
\hline 5 & & 1.5 & $12 \mathrm{~d}$ & 30 & & $55^{c}$ \\
\hline 6 & AgOTf & As solvent & $1 \mathrm{~min}$ & 100 & - & - \\
\hline 7 & $\mathrm{HgO}$ & As solvent & $8 \mathrm{~h}$ & 100 & - & - \\
\hline 8 & $\mathrm{HgBr}_{2}$ & As solvent & $1 \mathrm{~d}$ & 72 & 28 & - \\
\hline 9 & & 10 & $1 \mathrm{~d}$ & 41 & 33 & 26 \\
\hline 10 & & 5 & $1 \mathrm{~d}$ & 30 & 25 & $36^{c}$ \\
\hline 11 & & 1.5 & $1 \mathrm{~d}$ & 24 & 17 & $46^{c}$ \\
\hline 12 & $\mathrm{HgO}-\mathrm{HgBr}_{2}$ & As solvent & $16 \mathrm{~h}$ & 91 & 9 & - \\
\hline 13 & $\mathrm{Hg}(\mathrm{CN})_{2}$ & As solvent & $1 \mathrm{~d}$ & 84 & 8 & 8 \\
\hline 14 & & 10 & $1 \mathrm{~d}$ & 63 & 13 & $13^{c}$ \\
\hline 15 & & 5 & $1 \mathrm{~d}$ & 53 & 16 & $21^{\mathrm{c}}$ \\
\hline 16 & & 1.5 & $1 \mathrm{~d}$ & 52 & 10 & $23^{c}$ \\
\hline 17 & $\mathrm{InCl}_{3}$ & As solvent & $1 \mathrm{~d}$ & 83 & 17 & - \\
\hline 18 & & 10 & $1 \mathrm{~d}$ & 59 & 29 & 12 \\
\hline 19 & & 5 & $1 \mathrm{~d}$ & 71 & 14 & 15 \\
\hline 20 & & 1.5 & $1 \mathrm{~d}$ & 35 & 24 & $27^{c}$ \\
\hline
\end{tabular}

a Isolated yield.

b $C$-(2,3,4,6-Tetra-O-acetyl-1-fluoro- $\alpha$-D-galactopyranosyl)formamide was isolated in $\sim 3 \%$ yield from the mother liquor.

c Together with an unidentified product in $\sim 10-15 \%$ ratio. 
with $\mathrm{Hg}(\mathrm{CN})_{2}$ (entry 13) must be due to traces of water in the solvent. The structure of $\mathbf{3}$ was proven by NMR spectra showing characteristics similar to those of $\mathbf{2}$, except for the appearance of the $\mathrm{CN}$ resonance as a pseudo triplet type with $\sim 6 \mathrm{~Hz}$ coupling constants. Thus, the ${ }^{3} J_{\mathrm{H}-2, \mathrm{CN}}$ couplings for $\mathbf{2}$ and $\mathbf{3}$ support the gauche and the antiperiplanar arrangements, respectively, of the nuclei involved in the ${ }^{4} C_{1}$ conformation, and provide thereby unequivocal evidence for the anomeric configuration of these compounds. ${ }^{32-34}$

Having in mind the extension of this reaction to nitriles not applicable as solvents, several co-solvents were tried. In the presence of 5 equiv of $\mathrm{CH}_{3} \mathrm{CN}$ the transformation of $\mathbf{1}$ was either incomplete or gave complex product mixtures in $\mathrm{CH}_{2} \mathrm{Cl}_{2}, 1,4$-dioxane, HMPT, and benzene (in the light of their reactivity alcohols, esters, ketones, ${ }^{36}$ and sulfoxides ${ }^{36}$ were not considered as solvents). However, in $\mathrm{CH}_{3} \mathrm{NO}_{2}$ rather clean reactions were observed: with the mercury(II) salts and $\mathrm{InCl}_{3}$ (entries 9-11, 14-16, and 18-20, respectively) 2 and $\mathbf{3}$ were detected and, due to traces of water in the solvent $\mathrm{CH}_{3} \mathrm{NO}_{2}, 4$ also appeared in increasing ratios as the amount of $\mathrm{CH}_{3} \mathrm{CN}$ decreased. A conceivable anomerization of 2 to $\mathbf{3}$ was excluded by experiments conducted with pure $\mathbf{2}$ or $\mathbf{3}$ in $\mathrm{CH}_{3} \mathrm{NO}_{2}$ in the presence of 2 equiv of $\mathrm{HgBr}_{2}$ or $\mathrm{Hg}(\mathrm{CN})_{2}$ showing no change of these compounds even at elevated temperatures such as $100{ }^{\circ} \mathrm{C}$. On the other hand, with $\mathrm{Ag}_{2} \mathrm{CO}_{3}$ only 4 was observed as a by-product (entries 3-5). Thus, $\mathrm{CH}_{3} \mathrm{NO}_{2}$ and silver salts facilitate the use of nitriles in smaller excess maintaining the axial selectivity of the reaction.

Next the extension of the reaction to other nitriles applied as solvents and to starting C-(1-bromo-1-deoxy-D-glycopyranosyl)formamides $5^{37} \mathbf{6},,^{38}$ and $7^{35}$ besides $\mathbf{1}$ was investigated (Table 2). In these experiments $\mathrm{Ag}_{2} \mathrm{CO}_{3}$ was used because of the cost, stability, and easy handling of the reagent. The results show that under these conditions several 0 -acyl protected 1 -acylamino1-deoxy-D-glycopyranosyl cyanides 8-18 can be obtained in acceptable to good yields. In each compound the cyano group occupies an equatorial position as shown by the ${ }^{3} J_{\mathrm{H}-2, \mathrm{CN}}$ couplings in the range of $2.2-3.2 \mathrm{~Hz}$ indicating the incorporation of the nitrile reagent in an axial direction.

This Ritter-type reaction was also investigated with $N$-substituted $C$-(2,3,4,6-tetra- $O$-acetyl-1-bromo-1-deoxy- $\beta$-D-galactopyranosyl)formamides $\mathbf{1 9}^{32}$ and $\mathbf{2 0}$ (Scheme 2). Compound $\mathbf{2 0}$ was prepared from pentachlorophenyl $C$-(2,3,4,6-tetra-O-acetyl-1-bromo-1-deoxy- $\beta$-D-galactopyranosyl)formate and L-alanine methylester according to the procedure described for $\mathbf{1 9}^{32}$ Bromides 19 and 20 were each reacted with 1 equiv of $\mathrm{Ag}_{2} \mathrm{CO}_{3}$ in $\mathrm{CH}_{3} \mathrm{CN}$ as the solvent to give the spiro-oxazoline derivatives 21 and 23 in good and modest yield, respectively. The reaction of $\mathbf{1 9}$ with 10 equiv of the solid benzyloxycarbonylamino-acetonitrile was performed in $\mathrm{CH}_{3} \mathrm{NO}_{2}$ in the presence of 1 equiv of $\mathrm{Ag}_{2} \mathrm{CO}_{3}$ to give the spiro compound 22.

The structure of compounds 21-23 was established by NMR methods. The absence of exchangeable protons as well as resonances characteristic for imidate type carbons (155.8-158.2 ppm) were in accordance with the spirocyclic structures. Spectra for 22 exhibited two series of resonances indicating the presence of $E / Z$ isomers along the $\mathrm{C}-5=\mathrm{N}$ bond, while for $\mathbf{2 1}$ and $\mathbf{2 3}$ only one isomer was present. This configurational issue was not investigated further. The ${ }^{4} C_{1}$ conformation of the sugar rings was unequivocally assigned with the use of vicinal proton-proton coupling constants (see Section 3). The configuration of the anomeric carbon was established on the basis of three-bond heteronuclear couplings between $\mathrm{H}-2$ and the exocyclic imine type carbon $\mathrm{C}-5$ attached to $\mathrm{C}-1$ of the sugar part (parent monosaccharide numbering). These couplings were measured using a sensitivity enhanced gradient longrange ${ }^{13} \mathrm{C}-{ }^{1} \mathrm{H}$ correlation experiment (G-HSQMBC), ${ }^{39}$ and values around $3 \mathrm{~Hz}$ indicated gauche arrangement of the relevant atoms in the ${ }^{4} C_{1}$ conformation.

Table 2

Preparation of $O$-peracylated $N$-acyl-1-cyano-D-glycopyranosylamines

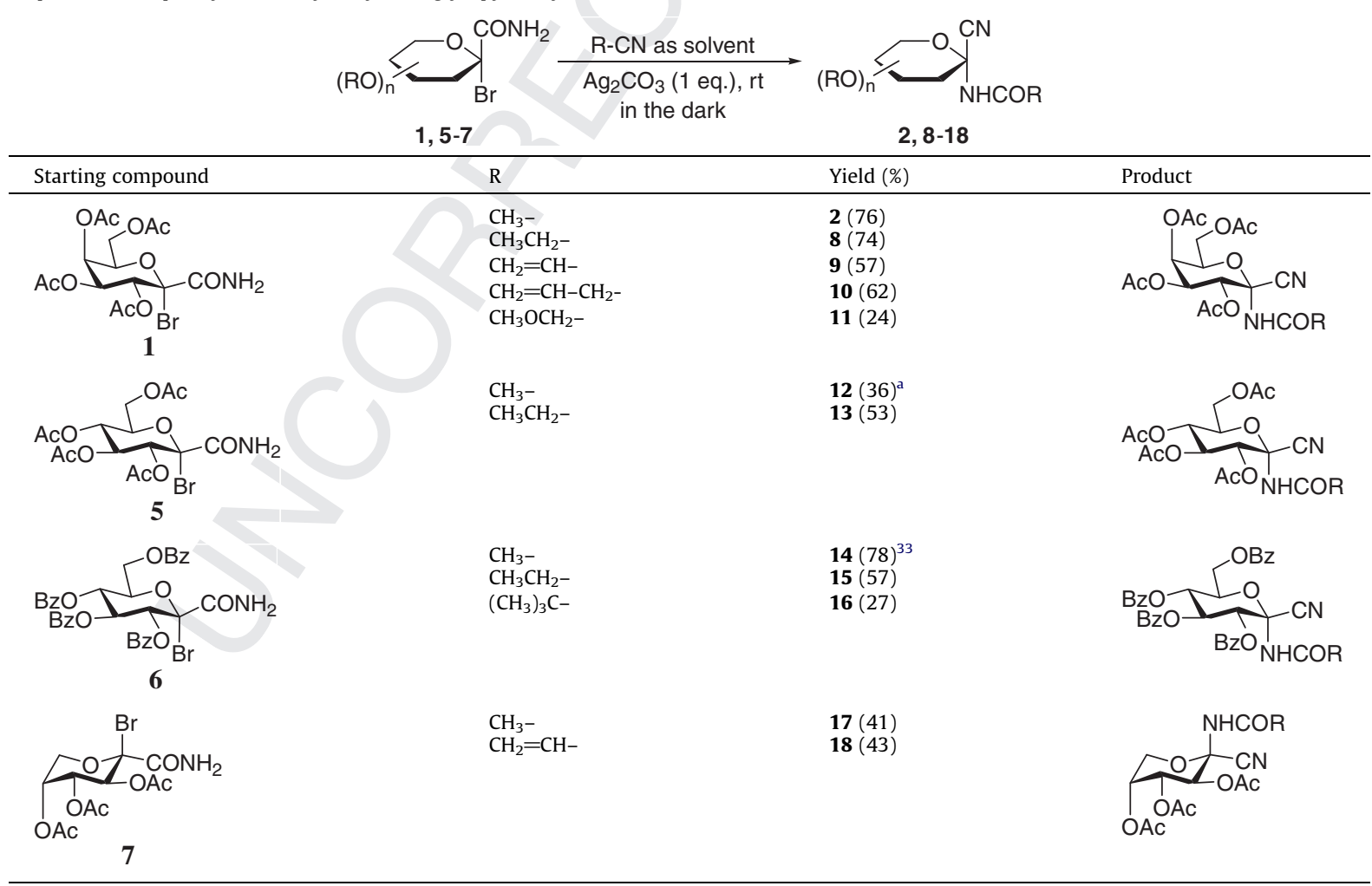

a With 2 equiv AgF. 


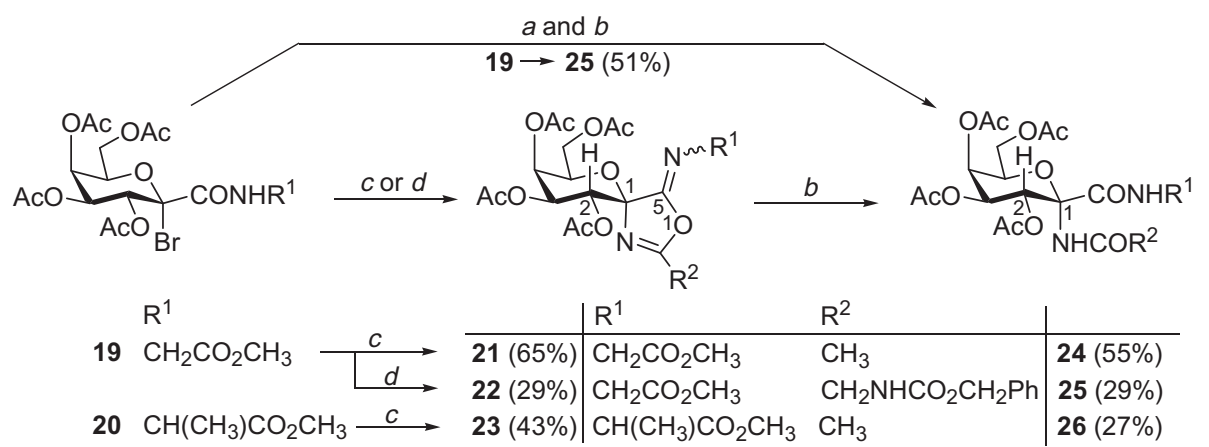

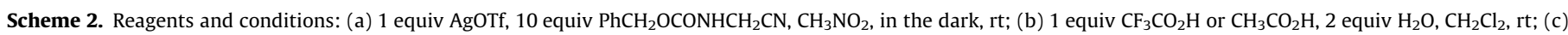
1 equiv $\mathrm{Ag}_{2} \mathrm{CO}_{3}, \mathrm{CH}_{3} \mathrm{CN}$, in the dark, rt; (d) 1 equiv $\mathrm{Ag}_{2} \mathrm{CO}_{3}, 10$ equiv $\mathrm{PhCH}_{2} \mathrm{OCONHCH}_{2} \mathrm{CN} \mathrm{CH}_{3} \mathrm{NO}_{2}$, in the dark, rt.

Spiro-oxazolines 21-23 were opened up by mild acidic hydrolysis to peptide derivatives 24-26, respectively. Carrying out the Ritter-reaction of 19 with benzyloxycarbonylamino-acetonitrile and the hydrolysis of the intermediate spiro-derivative in a continuous operation improved the overall yield of $\mathbf{2 5}$ to $51 \%$ for the two steps.

For 24-26 the NMR structural elucidation showed the presence of amide carbonyls (166.1-167.3 ppm) instead of imidate type carbons. The ${ }^{3} J_{\mathrm{H}-2, \mathrm{CONHR} 1}$ couplings were obtained from experiments as above, and the $2.4-2.9 \mathrm{~Hz}$ values were indicative of the equatorial position of the CONHR ${ }^{1}$ moiety. To corroborate this configurational assignment dipeptide $\mathbf{2 9}$, the anomeric pair of $\mathbf{2 4}$, was also synthesised (Scheme 3). Azide $\mathbf{2 7}^{32}$ was reduced by Raney-Ni to glycosylamine 28. In this reaction the formation of anomers or anomerisation of the formed glycosylamine cannot be excluded, however, the product $\mathbf{2 8}$ isolated from the crude mixture by crystallization existed in the $\beta$-D-anomeric configuration as revealed by the ${ }^{3} \mathrm{~J}_{\mathrm{H}-2, \mathrm{CONHR} 1}$ of $5.8 \mathrm{~Hz}$ coupling. Conventional acetylation of $\mathbf{2 8}$ by $\mathrm{AcCl}$ in pyridine gave a mixture of $\mathbf{2 4}$ and $\mathbf{2 9}$ (possibly due to anomerisation of $\mathbf{2 8}$ under the reaction conditions) which could be separated by column chromatography. For 29 the ${ }^{3} J_{\mathrm{H}-2, \mathrm{CONHR}}$ coupling was $5.3 \mathrm{~Hz}$, and thus these heteronuclear three-bond coupling values for the anomeric pairs $\mathbf{2 4}$ and $\mathbf{2 9}$ proved the configuration of the anomeric carbon in both compounds.

The formation of the new compounds by nitrile incorporation can be explained following the mechanistic proposal shown in Scheme 4. A promoter facilitates removal of a bromide ion from the substrates $(\mathbf{1}, \mathbf{5}-\mathbf{7}, \mathbf{1 9}, \mathbf{2 0})$ to give glycosylium ion $\mathbf{B}$. Axial attack of a nitrile may give glycosyl-nitrilium ion represented by resonance forms $\mathbf{F}$ and $\mathbf{G}$. Intramolecular nucleophilic attack by the amide carbonyl oxygen may lead to spirocyclic intermediate $\mathbf{H}$ which, on losing a proton, gives spiro-oxazolines 21-23 as well as $\mathbf{D}$. Tautomeric ring opening of the oxazoline in $\mathbf{D}$ and a subse- quent tautomerization of $\mathbf{C}$ result in the end-products $\mathbf{2}$, and $\mathbf{8}$ 18. In the presence of promoters other than silver salts formation of $\mathbf{3}$ was also observed. Since the anomeric interconversion of $\mathbf{2}$ and $\mathbf{3}$ was excluded, this can be accounted for by neighbouring group participation of the acyl protecting groups of $0-2$ as illustrated by $\mathbf{E}$ resulting in an equatorial attack of the nitrile. Glycosyl-nitrilium ion A may then follow similar transformations as those starting with $\mathbf{G}$, and end up with $\mathbf{3}$ as the isolable product. We have no explanation for the finding that with silver salts no compounds of type $\mathbf{3}$ were formed.

In conclusion, a Ritter-type reaction of a series of $O$-peracylated C-(1-bromo-1-deoxy-D-glycopyranosyl)formamides and nitriles promoted by silver salts gave access to $N$-acyl-1-cyano-D-glycopyranosylamines which can be regarded as anomeric $\alpha$-amino acid derivatives $^{40-43}$ and new derivatives of artificial ketoses. ${ }^{44}$ The reaction was extended to $N$-substituted $C$-(1-bromo-1-deoxy- $\beta$-Dgalactopyranosyl)formamides which gave anomeric spiro-oxazoline derivatives. In each of these compounds the newly formed $\mathrm{C}-\mathrm{N}$ bond at the anomeric carbon was axially oriented in spite of the participating protecting groups at the equatorial $0-2$. Mild acidic hydrolysis of the spiro-oxazolines led to di- and tripeptides incorporating anomeric $\alpha$-amino acids, thereby complementing reported synthetic methods for this class of sugar-peptide derivatives. $^{45-50}$

\section{Experimental}

\subsection{General methods}

Melting points were measured in open capillary tubes or on a Kofler hot-stage and are uncorrected. Optical rotations were determined with a Perkin-Elmer 241 polarimeter at room temperature.

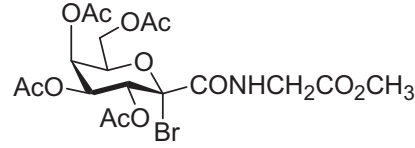

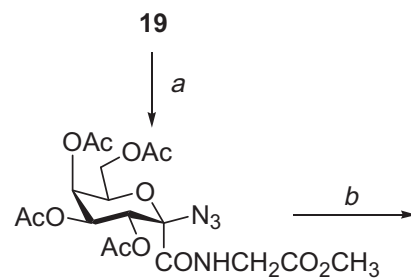

27 (65\%)

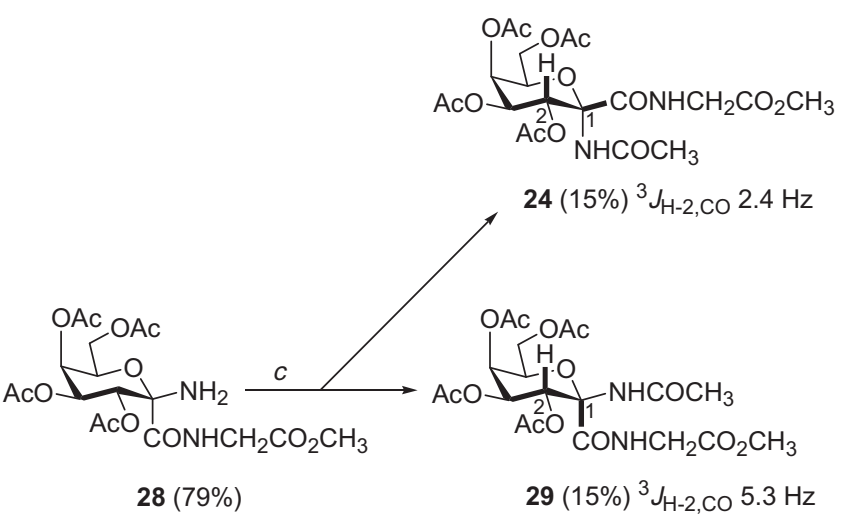

28 (79\%)
$29(15 \%){ }^{3} J_{\mathrm{H}-2, \mathrm{CO}} 5.3 \mathrm{~Hz}$

Scheme 3. Reagents and conditions: (a) 2 equiv $\mathrm{NaN}_{3}$, DMSO, rt; (b) $\sim 2$ equiv Raney- $\mathrm{Ni}, \mathrm{H}_{2}$, EtOAc, $70{ }^{\circ} \mathrm{C}$; (c) 2 equiv $\mathrm{CH}_{3} \mathrm{COCl}$, pyridine, rt. 


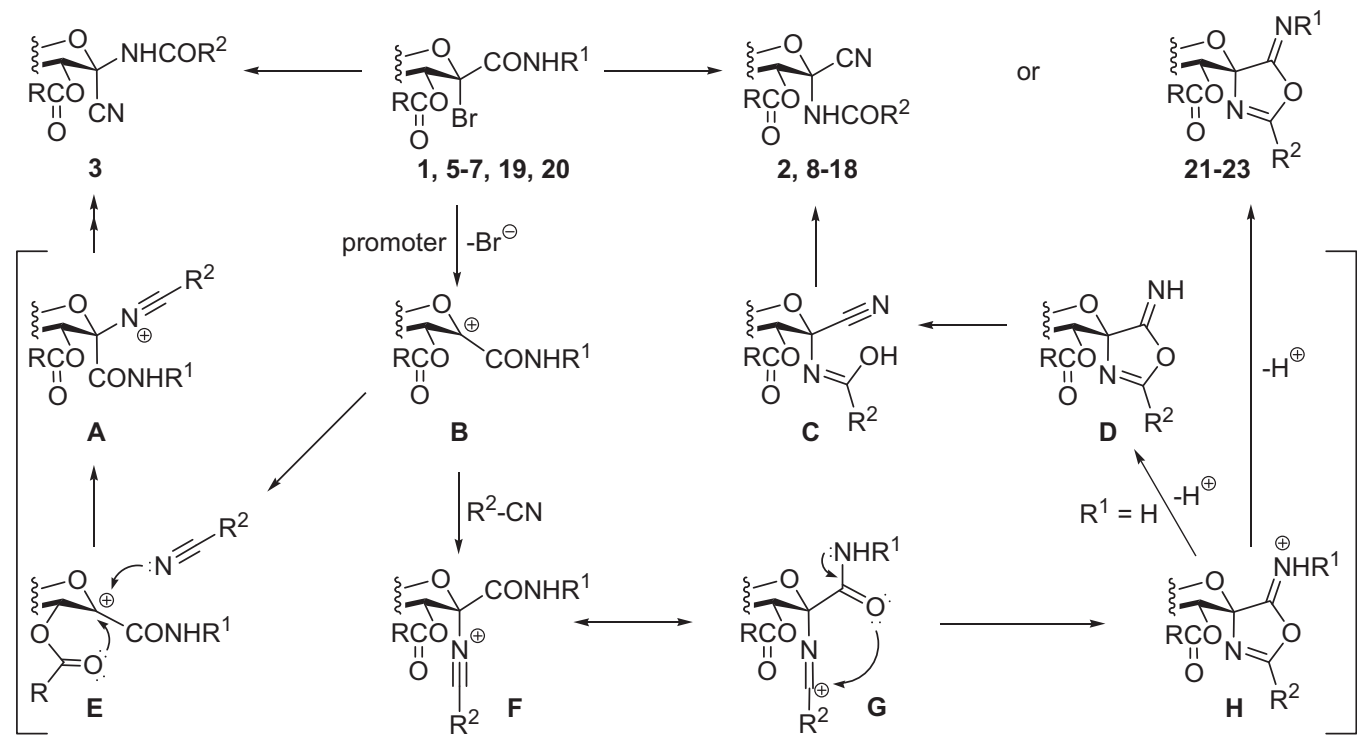

Scheme 4 .

NMR spectra were recorded with Bruker $200(200 / 50 \mathrm{MHz}$ for $\left.{ }^{1} \mathrm{H} /{ }^{13} \mathrm{C}\right)$, Bruker $360\left(360 / 90 \mathrm{MHz}\right.$ for $\left.{ }^{1} \mathrm{H} /{ }^{13} \mathrm{C}\right)$ or Avance DRX 500 $\left(500 / 125 \mathrm{MHz}\right.$ for $\left.{ }^{1} \mathrm{H} /{ }^{13} \mathrm{C}\right)$ spectrometers. Chemical shifts are referenced to Me4Si $\left({ }^{1} \mathrm{H}\right)$, or to the residual solvent signals $\left({ }^{13} \mathrm{C}\right)$. TLC was performed on DC-Alurolle Kieselgel $60 \mathrm{~F}_{254}$ (Merck), and the plates were visualised under UV light and by gentle heating. For column chromatography Kieselgel 60 (Merck, particle size 0.063$0.200 \mathrm{~mm}$ ) was used. Organic solutions were dried over anhydrous $\mathrm{MgSO}_{4}$ and concentrated under diminished pressure at $40-50{ }^{\circ} \mathrm{C}$ (water bath). $\mathrm{CH}_{3} \mathrm{CN}, \mathrm{CH}_{3} \mathrm{NO}_{2}$ were distilled from $\mathrm{P}_{2} \mathrm{O}_{5}$, other nitriles, AgOTf, and AgF were purchased from Sigma-Aldrich and used without further purification. $\mathrm{Ag}_{2} \mathrm{CO}_{3}$ was prepared from $\mathrm{AgNO}_{3}$ and $\mathrm{K}_{2} \mathrm{CO}_{3}$, and dried over $\mathrm{P}_{2} \mathrm{O}_{5}$.

\subsection{General procedure for the preparation of $\boldsymbol{O}$-peracylated} $\mathrm{N}$-acyl-1-cyano-D-glycopyranosylamines) (2-acylamino-2deoxy-hept(hex)-2-ulopyranosononitriles) $(2,8-18)$

An 0 -peracylated C-(1-bromo-1-deoxy-D-glycopyranosyl)formamide $\left(1,{ }^{28} 5,{ }^{37} \mathbf{6},{ }^{38}\right.$ or $\left.7,{ }^{35} 0.25 \mathrm{mmol}\right)$ was dissolved in a nitrile $(1 \mathrm{~mL})$, and silver carbonate $(0.07 \mathrm{~g}, 0.25 \mathrm{mmol})$ or silver fluoride $(0.063 \mathrm{~g}, 0.50 \mathrm{mmol})$ was added in one portion. The mixture was stirred at $\mathrm{rt}$ in the dark until complete disappearance of the starting material (2-3 days, TLC 3:1 EtOAc-hexane). It was then diluted with acetone $(9 \mathrm{~mL})$, filtered through a Celite pad, the filter cake was washed with acetone $(3 \mathrm{~mL})$, and the filtrate was concentrated. The residue was purified by column chromatography or crystallisation to give pure products.

\subsection{1. $N$-Acetyl-2,3,4,6-tetra- 0 -acetyl-1-cyano- $\alpha$-D-galactopyran- osylamine (2-acetamido-3,4,5,7-tetra-O-acetyl-2-deoxy- $\alpha$-D- galacto-hept-2-ulopyranosononitrile) (2)}

Prepared from $1(0.51 \mathrm{~g}, 1.12 \mathrm{mmol})$ in $\mathrm{CH}_{3} \mathrm{CN}$ according to Section 3.2. Reaction time $2 \mathrm{~d}$, purification by column chromatography $\left(1: 3\right.$ EtOAc- $\mathrm{CHCl}_{3}$ ), yield $0.35 \mathrm{~g}$ (76\%) white crystals. Mp: $155-156{ }^{\circ} \mathrm{C} ;[\alpha]_{\mathrm{D}}+49$ (c 1.15, $\left.\mathrm{CHCl}_{3}\right) ;{ }^{1} \mathrm{H}$ NMR $\left(\mathrm{CDCl}_{3}\right.$, $360 \mathrm{MHz}): \delta(\mathrm{ppm}) 7.90(\mathrm{~s}, 1 \hat{\mathrm{H}}, \mathrm{NH}), 5.75\left(\mathrm{~d}, 1 \mathrm{H}, J_{2,3} 10.7 \mathrm{~Hz}, \mathrm{H}-\right.$ 2), $5.30\left(\mathrm{dd}, 1 \mathrm{H} \mathrm{J} J_{2,3} 10.7 \mathrm{~Hz}, J_{3,4} 3.4 \mathrm{~Hz}, \mathrm{H}-3\right), 5.27$ (dd, $1 \mathrm{H}, J_{3,4}$ $\left.3.4 \mathrm{~Hz}, J_{4,5} 1.1 \mathrm{~Hz}, \mathrm{H}-4\right), 4.26\left(\mathrm{t}, 1 \mathrm{H}, J_{5,6} 6.0 \mathrm{~Hz}, J_{5,6^{\prime}} 6.0 \mathrm{~Hz}, \mathrm{H}-5\right)$, 4.10-4.08 (m, 2H, H-6, H-6'), 2.20, 2.19 (2), ' 2.03, 1.99 (4s, $\left.15 \mathrm{H}, \mathrm{OCOCH}_{3}, \mathrm{NHCOCH}_{3}\right) ;{ }^{13} \mathrm{C} \mathrm{NMR}\left(\mathrm{CDCl}_{3}, 90 \mathrm{MHz}\right): \delta(\mathrm{ppm})$ $171.2\left(\mathrm{NHCOCH}_{3}\right), 170.3,170.0,169.7168 .2$ (CO), 114.7 (CN, $\left.{ }^{3} \mathrm{~J}_{\mathrm{H}-2, \mathrm{CN}}=\sim 3.0\right), 77.7(\mathrm{C}-1), 67.7,67.5,67.3,66.7(\mathrm{C}-2-\mathrm{C}-5), 60.6$ (C-6), $22.9\left(\mathrm{NHCOCH}_{3}\right), 20.3(2), 20.5(2)\left(\mathrm{CH}_{3}\right)$; Anal. Calcd for $\mathrm{C}_{17} \mathrm{H}_{22} \mathrm{~N}_{2} \mathrm{O}_{10}$ (414.36): C, 49.28; $\mathrm{H}, 5.35 ; \mathrm{N}, 6.76$. Found: $\mathrm{C}$, 48.27; H, 5.43; N, 6.35.

3.2.2. $N$-Acetyl-2,3,4,6-tetra-O-acetyl-1-cyano- $\beta$-D-galactopyranosylamine (2-acetamido-3,4,5,7-tetra- 0 -acetyl-2-deoxy- $\beta$-Dgalacto-hept-2-ulopyranosononitrile) (3)

Compound $1(0.50 \mathrm{~g}, 1.10 \mathrm{mmol})$ was dissolved in a mixture of dry $\mathrm{CH}_{3} \mathrm{NO}_{2}$ (5 mL), $\mathrm{CH}_{3} \mathrm{CN}$ (10 equiv) was added, and the solution was stirred with freshly activated molecular sieves overnight. $\mathrm{HgBr}_{2}(0.50 \mathrm{~g}, 1.39 \mathrm{mmol})$ was added in one portion. The mixture was stirred at rt until complete disappearance of the starting material (TLC, 3:1 EtOAc-hexane) (2-3 days). It was then diluted with $\mathrm{CHCl}_{3}$, filtered through a Celite pad, and the filtrate was concentrated under diminished pressure. The residue was dissolved in $\mathrm{CHCl}_{3}$ and washed several times with $1 \mathrm{M} \mathrm{KBr}$ solution in order to remove mercury salts. The crude oil was purified by column chromatography $(1: 1 \rightarrow 3: 1$ EtOAc-hexane) to give pure products: $2(0.08 \mathrm{~g}, 20 \%)$ and 3 (0.15 g, 33\%), 4 (0.07 g, 15\%).

Compound 3 was obtained as white crystals. Mp $161-163{ }^{\circ} \mathrm{C}$; $[\alpha]_{\mathrm{D}}+262\left(\mathrm{c} 0.17, \mathrm{CHCl}_{3}\right) ;{ }^{1} \mathrm{H}$ NMR $\left(\mathrm{CDCl}_{3}, 360 \mathrm{MHz}\right): \delta(\mathrm{ppm})$ $7.21(\mathrm{~s}, 1 \mathrm{H}, \mathrm{NH}), 5.53$ (dd, $\left.1 \mathrm{H}, J_{3,4} 3.1 \mathrm{~Hz}, J_{4,5} 1.3 \mathrm{~Hz}, \mathrm{H}-4\right), 5.41$ (dd, 角, J $\left.J_{2,3} 10.5 \mathrm{~Hz}, J_{3,4} 3.1 \mathrm{~Hz}, \mathrm{H}-3\right), 5.22$ (d, $1 \mathrm{H}, J_{2,3} 10.5 \mathrm{~Hz}, \mathrm{H}-$ 2), $4.44\left(\mathrm{t}, 1 \mathrm{H}, J_{5,6} 6.8 \mathrm{~Hz}, J_{5,6^{\prime}} 6.8 \mathrm{~Hz}, \mathrm{H}-5\right), 4.21-4.16$ (m, 2H, H-6, $\left.\mathrm{H}-6^{\prime}\right), 2.21,2.16,2.06(2), \hat{2.01}\left(4 \mathrm{~s}, 15 \mathrm{H}, \mathrm{OCOCH}_{3}, \mathrm{NHCOCH}_{3}\right) ;{ }^{13} \mathrm{C}$ NMR $\left(\mathrm{CDCl}_{3}, 90 \mathrm{MHz}\right): \delta(\mathrm{ppm}) 17 \hat{1} .7\left(\mathrm{NHCOCH}_{3}\right), 170.1,169.6$, 169.3169 .1 (CO), $111.9\left(\mathrm{CN},{ }^{3} \mathrm{~J}_{\mathrm{H}-2, \mathrm{CN}}=\sim 5.8 \mathrm{~Hz}\right), 79.9(\mathrm{C}-1), 71.2$, 68.5 (2), 66.1 (C-2-C-5), 60.2 (C-6), $23.1\left(\mathrm{NHCOCH}_{3}\right), 20.5,20.4$, 20.3, $20.2\left(\mathrm{CH}_{3}\right)$; Anal. Calcd for $\mathrm{C}_{17} \mathrm{H}_{22} \mathrm{~N}_{2} \mathrm{O}_{10}$ (414.36): C, 49.28; H, 5.35; N, 6.76. Found: ${ }^{\mathrm{C}}, 48.77 ; \mathrm{H}, 5.23 ; \mathrm{N}, 6.00$.

\subsection{3. $N$-Propanoyl-2,3,4,6-tetra- $O$-acetyl-1-cyano- $\alpha$-D-galacto- pyranosylamine $(3,4,5,7$-tetra-O-acetyl-2-deoxy-2-propan- amido- $\alpha$-D-galacto-hept-2-ulopyranosononitrile) (8)}

Prepared from $1(0.05 \mathrm{~g}, 0.11 \mathrm{mmol})$ in $\mathrm{CH}_{3} \mathrm{CH}_{2} \mathrm{CN}$ according to Section 3.2. Reaction time $2 \mathrm{~d}$, purification by column chromatography ( $1: 3 \mathrm{EtOAc}-\mathrm{CHCl}_{3}$ ), yield $0.035 \mathrm{~g}$ (74\%) white crystals. Mp: $186-187{ }^{\circ} \mathrm{C} ;[\alpha]_{\mathrm{D}}+55\left(\mathrm{c} 0.91, \mathrm{CHCl}_{3}\right) ;{ }^{1} \mathrm{H} \mathrm{NMR}\left(\mathrm{CDCl}_{3}, 360 \mathrm{MHz}\right)$ : $\delta(\mathrm{ppm}) 7.68(\mathrm{~s}, 1 \mathrm{H}, \mathrm{NH}), 5.76\left(\mathrm{~d}, 1 \mathrm{H}, J_{2,3} 10.6 \mathrm{~Hz}, \mathrm{H}-2\right), 5.30(\mathrm{dd}$, $\left.1 \mathrm{H}, J_{2,3} 10.6 \mathrm{~Hz}, J_{3,4} 3.4 \mathrm{~Hz}, \mathrm{H}-3\right), 5.38$ (dd, $1 \mathrm{H}, J_{3,4} 3.4 \mathrm{~Hz}, J_{4,5}$ $1.0 \mathrm{~Hz}, \mathrm{H}-4), 4.26\left(\mathrm{t}, 1 \mathrm{H}, J_{5,6} 6.6 \mathrm{~Hz}, J_{5,6^{\prime}} 6.6 \mathrm{~Hz}, \mathrm{H}-5\right), 4.10(\mathrm{~m}, 2 \mathrm{H}$, 
H-6, H-6' ), 2.42 (q, 2H, J 7.3 Hz, J 7.3 Hz, $\mathrm{CH}_{2}$ ), 2.20, 2.17, 2.02, 1.98 $\left(4 \mathrm{~s}, 12 \mathrm{H}, \mathrm{OCOCH}_{3}\right), 1.21\left(\mathrm{t}, 3 \mathrm{H}, J 7.3 \mathrm{~Hz}, J 7.3 \mathrm{~Hz}, \mathrm{CH}_{3}\right) ;{ }^{13} \mathrm{C} \mathrm{NMR}$ $\left(\mathrm{CDCl}_{3}, 90 \mathrm{MHz}\right): \delta(\mathrm{ppm}) 174.5\left(\mathrm{NHCOCH}_{2} \mathrm{CH}_{3}\right), 170.3,170.0$, 169.7168 .1 (CO), $114.8\left(\mathrm{CN},{ }^{3} J_{\mathrm{H}-2, \mathrm{CN}}=\sim 3.0\right), 77.6(\mathrm{C}-1), 67.6(2)$, 67.4, 67.3, 66.7 (C-2-C-5), 60.6 (C-6), $28.9\left(\mathrm{NHCOCH}_{2} \mathrm{CH}_{3}\right), 20.3$ (2), 20.5 (2) $\left(\mathrm{CH}_{3}\right), 8.9 \quad\left(\mathrm{NHCOCH}_{2} \mathrm{CH}_{3}\right)$; Anal. Calcd for $\mathrm{C}_{18} \mathrm{H}_{24} \mathrm{~N}_{2} \mathrm{O}_{10}$ (428.39): C, 50.47; H, 5.65; N, 6.54. Found: C, 50.41; H, 5.93; N, 6.67.

\subsection{4. $N$-Propenoyl-2,3,4,6-tetra-O-acetyl-1-cyano- $\alpha$-D-galacto- pyranosylamine (3,4,5,7-tetra-O-acetyl-2-deoxy-2-propen- amido- $\alpha$-D-galacto-hept-2-ulopyranosononitrile) (9)}

Prepared from $1(0.05 \mathrm{~g}, 0.11 \mathrm{mmol})$ in $\mathrm{CH}_{2} \mathrm{CHCN}$ according to Section 3.2. Reaction time $2 \mathrm{~d}$, purification by column chromatography $\left(1: 3 \mathrm{EtOAc}-\mathrm{CHCl}_{3}\right)$, yield $0.03 \mathrm{~g}$ (57\%) white crystals. Mp: $158-160{ }^{\circ} \mathrm{C} ;[\alpha]_{\mathrm{D}}+61\left(\mathrm{c} 0.80, \mathrm{CHCl}_{3}\right) ;{ }^{1} \mathrm{H} \mathrm{NMR}\left(\mathrm{CDCl}_{3}, 360 \mathrm{MHz}\right)$ : $\delta(\mathrm{ppm}) 7.20(\mathrm{~s}, 1 \mathrm{H}, \mathrm{NH}), 6.51\left(\mathrm{~d}, 1 \mathrm{H}, J 7.3 \mathrm{~Hz}, \mathrm{CH}_{2}\right), 6.29(\mathrm{dd}, 1 \mathrm{H}$, J $7.3 \mathrm{~Hz}, J 6.9 \mathrm{~Hz}, \mathrm{CH}), 5.86\left(\mathrm{~d}, 1 \mathrm{H}, J 6.9 \mathrm{~Hz}, \mathrm{CH}_{2}\right), 5.78\left(\mathrm{~d}, 1 \mathrm{H}, J_{2,3}\right.$ $10.6 \mathrm{~Hz}, \mathrm{H}-2$ ), 5.25 (dd, $1 \mathrm{H}, J_{2,3} 10.6 \mathrm{~Hz}, J_{3,4} 2.8 \mathrm{~Hz}, \mathrm{H}-3$ ), 5.39 (dd, $\left.1 \mathrm{H}, J_{3,4} 2.8 \mathrm{~Hz}, J_{4,5} 1.1 \mathrm{~Hz}, \mathrm{H}-4\right), 4.23\left(\mathrm{t}, 1 \mathrm{H}, J_{5,6} 6.6 \mathrm{~Hz}, J_{5,6^{\prime}} 6.6 \mathrm{~Hz}\right.$, $\mathrm{H}-5$ ), 4.18 (dd, $1 \mathrm{H}, J_{6,6^{\prime}} 10.7 \mathrm{~Hz}, J_{5,6} 6.6 \mathrm{~Hz}, \mathrm{H}-6$ ), 4.09 (dd, $1 \mathrm{H}, J_{6,6^{\prime}}$ $\left.10.7 \mathrm{~Hz}, J_{5,6} 6.6 \mathrm{~Hz}, \mathrm{H}-6\right), 2.20,2.16,2.02,1.99$ (4s, 12H, $\mathrm{OCOCH}_{3}$ ); ${ }^{13} \mathrm{C} \mathrm{NMR}\left(\mathrm{CDCl}_{3}, 90 \mathrm{MHz}\right): \delta(\mathrm{ppm}) 170.3\left(\mathrm{NHCOCH}=\mathrm{CH}_{2}\right), 170.1$, 170.0, 169.7, 168.1 (CO), $130.2\left(\mathrm{CH}=\mathrm{CH}_{2}\right), 128.8\left(\mathrm{CH}=\mathrm{CH}_{2}\right), 114.5$ $\left(\mathrm{CN},{ }^{3} \widehat{\mathrm{J}}-2, \mathrm{CN}=\sim 3.2\right), 78.2(\mathrm{C}-1), 68.1,67.7,67.6,66.7(\mathrm{C}-2-\mathrm{C}-5)$, 60.6 (C-6), 20.5 (2), 20.4 (2) $\left(\mathrm{CH}_{3}\right)$; Anal. Calcd for $\mathrm{C}_{18} \mathrm{H}_{22} \mathrm{~N}_{2} \mathrm{O}_{10}$ (426.37): C, 50.70; H, 5.20; N, 6.57. Found: C, 49.93; H, 5.12; N, 6.39 .

3.2.5. $N$-(But-3-enoyl)-2,3,4,6-tetra-O-acetyl-1-cyano- $\alpha$-D-galactopyranosylamine (3,4,5,7-tetra-O-acetyl-2-(but-3-enamido)-2deoxy- $\alpha$-D-galacto-hept-2-ulopyranosononitrile) (10)

Prepared from 1 ( $0.10 \mathrm{~g}, 0.22 \mathrm{mmol})$ in $\mathrm{CH}_{2} \mathrm{CHCH}_{2} \mathrm{CN}$ according to Section 3.2. Reaction time $2 \mathrm{~d}$, purification by column chromatography $\left(\hat{1}: 3\right.$ EtOAc- $\left.\mathrm{CHCl}_{3}\right)$, yield $0.06 \mathrm{~g}(62 \%)$ white crystals. Mp: $149-150{ }^{\circ} \mathrm{C} ;[\alpha]_{\mathrm{D}}+57\left(\mathrm{c} 0.88, \mathrm{CHCl}_{3}\right) ;{ }^{1} \mathrm{H}$ NMR $\left(\mathrm{CDCl}_{3}\right.$, $360 \mathrm{MHz}): \delta(\mathrm{ppm}) 6.85(\mathrm{~s}, 1 \mathrm{H}, \mathrm{NH}), 5.99(\mathrm{~m}, 1 \mathrm{H}, \mathrm{CH}), 5.38(\mathrm{~d}$, $\left.1 \mathrm{H}, J_{2,3} 10.7 \mathrm{~Hz}, \mathrm{H}-2\right), 5.40-5.35$ (m, 2H, H-3, H-4), 5.14 (m, 2H, $\left.\mathrm{CH}_{2}\right), 4.20\left(\mathrm{~m}, 3 \mathrm{H}, \mathrm{H}-5, \mathrm{H}-6, \mathrm{H}-6^{\prime}\right), 3.19\left(\mathrm{~d}, 2 \mathrm{H}, J 7.3 \mathrm{~Hz}, \mathrm{CH}_{2}\right)$, $2.20,2.16,2.03,2.00\left(4 \mathrm{~s}, \hat{2} \mathrm{H}, \mathrm{OCOCH}_{3}\right) ;{ }^{13} \mathrm{C} \mathrm{NMR}\left(\mathrm{CDCl}_{3}\right.$, $90 \mathrm{MHz}): \delta(\mathrm{ppm}) 170.3\left(\mathrm{NHCOCH}_{2} \mathrm{CH}=\mathrm{CH}_{2}\right), 170.0,169.9167 .8$, $167.7(\mathrm{CO}), 129.9\left(\mathrm{NHCOCH}_{2} \mathrm{CH}=\mathrm{CH}_{2}\right), 120.5\left(\mathrm{NHCOCH}_{2} \mathrm{CH}=\mathrm{CH}_{2}\right)$, $114.4\left(\mathrm{CN},{ }^{3} \mathrm{~J}_{\mathrm{H}-2, \mathrm{CN}}=\sim 3.1 \mathrm{~Hz}\right), \overline{7} 8.0(\mathrm{C}-1), 68.0,67.7,67.6,66.6(\mathrm{C}-$ 2-C-5), 60.7 (C-6), $41.0\left(\mathrm{NHCOCH}_{2} \mathrm{CH}=\mathrm{CH}_{2}\right) 20.5$ (2), 20.4, 20.3

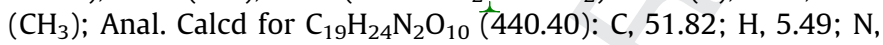
6.36. Found: C, 51.34; H, 5.43; N, 6.55.

3.2.6. $N$-Methoxyacetyl-2,3,4,6-tetra- 0 -acetyl-1-cyano- $\alpha$-D-galactopyranosylamine (3,4,5,7-tetra-0-acetyl-2-deoxy-2-methoxyacetamido- $\alpha$-D-galacto-hept-2-ulopyranosononitrile) (11)

Prepared from $1(0.10 \mathrm{~g}, 0.22 \mathrm{mmol})$ in $\mathrm{CH}_{3} \mathrm{OCH}_{2} \mathrm{CN}$ according to Section 3.2. Reaction time $2 \mathrm{~d}$, purification by column chromatography ( $1: 3$ EtOAc- $\mathrm{CHCl}_{3}$ ), yield $0.02 \mathrm{~g}$ (24\%) white crystals. Mp: $149-151{ }^{\circ} \mathrm{C} ;[\alpha]_{\mathrm{D}}+29\left(\mathrm{c} 1.22, \mathrm{CHCl}_{3}\right) ;{ }^{1} \mathrm{H} \mathrm{NMR}\left(\mathrm{CDCl}_{3}\right.$, $360 \mathrm{MHz}): \delta(\mathrm{ppm}) 7.34(\mathrm{~s}, 1 \mathrm{H}, \mathrm{NH}), 5.78\left(\mathrm{~d}, 1 \mathrm{H}, J_{2,3} 10.9 \mathrm{~Hz}, \mathrm{H}-\right.$ 2), $5.41\left(\mathrm{dd}, 1 \mathrm{H}, J_{3,4} 3.2 \mathrm{~Hz}, J_{4,5} 1.1 \mathrm{~Hz}, \mathrm{H}-4\right), 5.11$ (dd, $1 \mathrm{H}, J_{2,3}$ $10.9 \mathrm{~Hz}, J_{3,4} 3.2 \mathrm{~Hz}, \mathrm{H}-3$ ), $4.22-4.05$ (m, 3H, H-5, H-6, H-6'), 4.04 $\left(\mathrm{d}, 1 \mathrm{H}, J 6.9 \mathrm{~Hz}, \mathrm{CH}_{2}\right), 3.97\left(\mathrm{~d}, 1 \mathrm{H}, J 6.9 \mathrm{~Hz}, \mathrm{CH}_{2}\right), 3.49(\mathrm{~s}, 3 \mathrm{H}$, $\left.\mathrm{OCH}_{3}\right), 2.21,2.17,2.03,2.01\left(4 \mathrm{~s}, 12 \mathrm{H}, \mathrm{OCOCH}_{3}\right) ;{ }^{13} \mathrm{C} \mathrm{NMR}\left(\mathrm{CDCl}_{3}\right.$, $90 \mathrm{MHz}): \delta(\mathrm{ppm}) 170.4\left(\mathrm{NHCOCH}_{2} \mathrm{OCH}_{3}\right), 170.1,169.9169 .3$, $167.7(\mathrm{CO}), 114.1\left(\mathrm{CN},{ }^{3} \mathrm{~J}_{\mathrm{H}-2, \mathrm{CN}}=\sim 3.0\right), 77.8(\mathrm{C}-1), 71.6$ (NHCO$\left.\mathrm{CH}_{2} \mathrm{OCH}_{3}\right), 68.1,67.8,67.5,66.4$ (C-2-C-5), 60.5 (C-6), 59.3 (NHC$\left.\mathrm{OCH}_{2} \mathrm{OCH}_{3}\right), 20.6$ (2), 20.4, $20.3\left(\mathrm{CH}_{3}\right)$; Anal. Calcd for $\mathrm{C}_{18} \mathrm{H}_{24} \mathrm{~N}_{2} \mathrm{O}_{11}$ (444.39): C, 48.65; H, 5.44; N, 6.30. Found: C, 50.00; $\mathrm{H}, 5.70 ; \mathrm{N}, 6.47$.
3.2.7. $N$-Acetyl-2,3,4,6-tetra-O-acetyl-1-cyano- $\alpha$-D-glucopyranosylamine (2-acetamido-3,4,5,7-tetra- 0 -acetyl-2-deoxy- $\alpha$-Dgluco-hept-2-ulopyranosononitrile) (12)

Prepared from 5 ( $0.60 \mathrm{~g}, 1.32 \mathrm{mmol})$ in $\mathrm{CH}_{3} \mathrm{CN}$ with $\mathrm{AgF}$ according to Section 3.2. Reaction time $1 \mathrm{~d}$, purification by column chromatography $(1: 1 \rightarrow 3: 1$ EtOAc-hexane), yield $0.20 \mathrm{~g}$ (36\%) white crystals. Mp $179-181^{\circ} \mathrm{C} ;[\alpha]_{\mathrm{D}}+57$ (c 1.01, acetone).

${ }^{1} \mathrm{H}$ NMR $\left(\left(\mathrm{CD}_{3}\right)_{2} \mathrm{CO}, 200 \mathrm{MHz}\right): \delta(\mathrm{ppm}) 8.72(\mathrm{~s}, 1 \mathrm{H}, \mathrm{NH}), 5.52(\mathrm{~d}$, $\left.1 \mathrm{H}, J_{2,3} 10.0 \mathrm{~Hz}, \mathrm{H}-2\right), 5.42\left(\mathrm{dd}, 1 \mathrm{H}, J_{3,4} 4.5 \mathrm{~Hz}, J_{4,5} 2.4 \mathrm{~Hz}, \mathrm{H}-4\right), 5.16$ (dd, 1H, J2,3 $9.8 \mathrm{~Hz}, J_{3,4} 4.5 \mathrm{~Hz}, \mathrm{H}-3$ ), 4.31 (dd, 1H, J $J_{6,6} 12.3 \mathrm{~Hz}, J_{5,6}$ $6.0 \mathrm{~Hz}, \mathrm{H}-6), 4.02$ (dd, $\left.1 \mathrm{H}, J_{6,6^{\prime}} 12.3 \mathrm{~Hz}, J_{5,6} 6.0 \mathrm{~Hz}, \mathrm{H}_{-} 6^{\prime}\right), 2.11$, $2.10,2.02,1.99,1.96\left(5 \mathrm{~s}, 15 \mathrm{H}, \mathrm{NHCOCH}_{3}, \mathrm{OCOCH}_{3}\right) ;{ }^{13} \mathrm{C} \mathrm{NMR}$ $\left(\left(\mathrm{CD}_{3}\right)_{2} \mathrm{CO}, 50 \mathrm{MHz}\right): \delta(\mathrm{ppm}) 170.7\left(\mathrm{NHCOCH}_{3}\right), 170.4,170.2$, 169.8, $168.9(\mathrm{CO}), 115.9\left(\mathrm{CN},{ }^{3} J_{\mathrm{H}-2, \mathrm{CN}}=\sim 3.0 \mathrm{~Hz}\right), 78.1(\mathrm{C}-1), 71.5$, 71.1, 69.2, 68.5 (C-2-C-5), 61.8 (C-6), $22.9\left(\mathrm{NHCOCH}_{3}\right), 20.5,20.4$ (2), 20.3 $\left(\mathrm{CH}_{3}\right)$; Anal. Calcd for $\mathrm{C}_{17} \mathrm{H}_{22} \mathrm{~N}_{2} \mathrm{O}_{10}$ (414.364): C, 49.28; H, 5.35; N, 6.76. Found: C, 48.52; H, 5.60; N, 6.70.

3.2.8. $N$-Propanoyl-2,3,4,6-tetra-O-acetyl-1-cyano- $\alpha$-D-glucopyranosylamine (3,4,5,7-tetra-O-acetyl-2-deoxy-2-propanamido- $\alpha$-D-gluco-hept-2-ulopyranosononitrile) (13)

Prepared from $5(0.31 \mathrm{~g}, 0.69 \mathrm{mmol})$ in $\mathrm{CH}_{3} \mathrm{CH}_{2} \mathrm{CN}$ according to Section 3.2. Reaction time $3 \mathrm{~d}$, purification by column chromatography $\left(1: 3 \mathrm{EtOAc}-\mathrm{CHCl}_{3}\right)$, yield $0.16 \mathrm{~g}(53 \%)$ white crystals from EtOAc. Mp: $187-189{ }^{\circ} \mathrm{C} ;[\alpha]_{\mathrm{D}}+25\left(\mathrm{c} 0.61, \mathrm{CHCl}_{3}\right) ;{ }^{1} \mathrm{H}$ NMR $\left(\mathrm{CDCl}_{3}\right.$, $200 \mathrm{MHz}): \delta(\mathrm{ppm}) 6.97(\mathrm{~s}, 1 \mathrm{H}, \mathrm{NH}), 5.57\left(\mathrm{~d}, 1 \mathrm{H}, J_{2,3} 9.8 \mathrm{~Hz}, \mathrm{H}-2\right)$, 5.31 (dd, $\left.1 \mathrm{H}, J_{3,4} 4.4 \mathrm{~Hz}, J_{4,5} 1.1 \mathrm{~Hz}, \mathrm{H}-4\right), 5.16$ (dd, $1 \mathrm{H}, J_{2,3} 9.8 \mathrm{~Hz}$, $J_{3,4} 4.4 \mathrm{~Hz}, \mathrm{H}-3$ ), 4.29 (dd, $1 \mathrm{H}, J_{6,6^{\prime}} 12.5 \mathrm{~Hz}, J_{5,6} 6.0 \mathrm{~Hz}, \mathrm{H}-6$ ), 4.00 (dd, $\left.1 \mathrm{H}, J_{6,6^{\prime}} 12.5 \mathrm{~Hz}, J_{5,6} 6.0 \mathrm{~Hz}, \mathrm{H}-6\right), 3.96\left(\mathrm{t}, 1 \mathrm{H}, J_{5,6} 6.0 \mathrm{~Hz}, J_{5,6^{\prime}}\right.$ $6.0 \mathrm{~Hz}, \mathrm{H}-5), 2.42$ (q, $\left.2 \mathrm{H}, J 7.3 \mathrm{~Hz}, J 7.2 \mathrm{~Hz}, \mathrm{CH}_{2} \mathrm{CH}_{3}\right), 2.16,2.11$, 2.03 (2) (3s, 12H, $\left.\mathrm{OCOCH}_{3}\right), 1.21$ (t, 3H, J 7.3 Hz, J 7.2 Hz, $\mathrm{CH}_{2} \mathrm{CH}_{3}$ ); ${ }^{13} \mathrm{C} \mathrm{NMR}\left(\mathrm{CDCl}_{3}, 50 \mathrm{MHz}\right): \delta(\mathrm{ppm}) 173.4\left(\mathrm{NHCOCH}_{2} \mathrm{CH}_{3}\right), 170.3$, 169.8, 168.7, 167.6 (CO), $114.1\left(\mathrm{CN},{ }^{3} J_{\mathrm{H}-2, \mathrm{CN}}=\sim 3.0 \mathrm{~Hz}\right), 76.0(\mathrm{C}-1)$, 70.1, 69.8, 68.2, 66.9 (C-2-C-5), 60.6 (C-6), $28.7\left(\mathrm{CH}_{2} \mathrm{CH}_{3}\right), 20.2$, 20.1, 20.0, $19.9\left(\mathrm{CH}_{3}\right), 8.4\left(\mathrm{CH}_{2} \mathrm{CH}_{3}\right)$; Anal. Calcd for $\mathrm{C}_{18} \mathrm{H}_{24} \mathrm{~N}_{2} \mathrm{O}_{10}$ (428.391): C, 50.47; H, 5.65; N, 6.54. Found: C, 50.03; H, 5.52; N, 6.23.

3.2.9. $N$-Propanoyl-2,3,4,6-tetra- $O$-benzoyl-1-cyano- $\alpha$-D-glucopyranosylamine (3,4,5,7-tetra-0-benzoyl-2-deoxy-2-propanamido- $\alpha$-D-gluco-hept-2-ulopyranosononitrile) (15)

Prepared from 6 ( $0.30 \mathrm{~g}, 0.43 \mathrm{mmol})$ in $\mathrm{CH}_{3} \mathrm{CH}_{2} \mathrm{CN}$ according to Section 3.2. Reaction time $2 \mathrm{~d}$, purification by column chromatography $\left(1: 3\right.$ EtOAc- $\left.\mathrm{CH}_{2} \mathrm{Cl}_{2}\right)$, yield $0.17 \mathrm{~g}$ (57\%) white crystals. Mp: 239-241 ${ }^{\circ} \mathrm{C} ;[\alpha]_{\mathrm{D}}+62.0\left(\mathrm{c} 1.03, \mathrm{CHCl}_{3}\right) ;{ }^{1} \mathrm{H}$ NMR $\left(\mathrm{CDCl}_{3}\right.$, $360 \mathrm{MHz}): \delta(\mathrm{ppm}): 8.00-7.08(\mathrm{~m}, 20 \mathrm{H}, \operatorname{ArH}), 7.71(\mathrm{~s}, 1 \mathrm{H}, \mathrm{NH})$, 6.88 (pseudo t, $1 \mathrm{H}, J 9.8 \mathrm{~Hz}, J 9.2 \mathrm{~Hz}, \mathrm{H}-3$ or $\mathrm{H}-4), 6.36$ (d, $1 \mathrm{H}, J_{2,3}$ $9.8 \mathrm{~Hz}, \mathrm{H}-2$ ), 5.82 (pseudo t, $1 \mathrm{H}, J 9.8 \mathrm{~Hz}, J 9.2 \mathrm{~Hz}, \mathrm{H}-3$ or $\mathrm{H}-4$ ), 4.46-4.40 (m, 3H, H-5, H-6, H-6'), 2.40 (pseudo q, $2 \mathrm{H}, J 7.2 \mathrm{~Hz}, J$ $5.2 \mathrm{~Hz}, \mathrm{CH}_{2}$ ), 1.10 (pseudo t, $\widehat{3} \mathrm{H}, J 7.2 \mathrm{~Hz}, J 5.2 \mathrm{~Hz}, \mathrm{CH}_{3}$ ); ${ }^{13} \mathrm{C} \mathrm{NMR}$ $\left(\mathrm{CDCl}_{3}, 90 \mathrm{MHz}\right): \delta(\mathrm{ppm}): 166.1\left(\mathrm{NHCOCH}_{2} \mathrm{CH}_{3}\right), 166.1,165.8$, 164.8, 164.7 (CO), 134.0-127.4 (benzoyl ArC), $114.8\left(\mathrm{CN},{ }^{3} \mathrm{~J}_{\mathrm{H}-}\right.$ $2, \mathrm{CN},=\sim 2.2 \mathrm{~Hz}$ ), 77.8 (C-1), 71.4, 70.8, 69.2, 68.5 (C-2-C-5), 62.0 (C-6), $29.0\left(\mathrm{NHCOCH}_{2} \mathrm{CH}_{3}\right), 8.10\left(\mathrm{NHCOCH}_{2} \mathrm{CH}_{3}\right)$; Anal. Calcd for $\mathrm{C}_{38} \mathrm{H}_{32} \mathrm{~N}_{2} \mathrm{O}_{10}$ (676.69): C, 67.45; H, 4.77; N, 4.14. Found: C, 67.10; $\mathrm{H}, 4.36$; N, 4.01 .

\subsubsection{0. $N$-Pivaloyl-2,3,4,6-tetra-O-benzoyl-1-cyano- $\alpha$-D-gluco- pyranosylamine $(3,4,5,7$-tetra-O-benzoyl-2-deoxy-2-pival- amido- $\alpha$-D-gluco-hept-2-ulopyranosononitrile) (16)}

Prepared from $6(0.20 \mathrm{~g}, 0.28 \mathrm{mmol})$ in $\left(\mathrm{CH}_{3}\right)_{3} \mathrm{CCN}$ according to Section 3.2. Reaction time $2 \mathrm{~d}$, purification by column chromatography (1:2 EtOAc-Hexane), yield $0.06 \mathrm{~g}$ (27\%) white crystals and compound $4(0.05 \mathrm{~g}, 27 \%)$. Mp: $226-229^{\circ} \mathrm{C} ;[\alpha]_{\mathrm{D}}+37$ (c 0.98, $\left.\mathrm{CHCl}_{3}\right) ;{ }^{1} \mathrm{H} \mathrm{NMR}\left(\mathrm{CDCl}_{3}, 360 \mathrm{MHz}\right): \delta(\mathrm{ppm}): 8.10-7.24(\hat{\mathrm{m}}, 20 \mathrm{H}$, ArH), 6.85 (s, 1H, NH), 6.13 (d, $1 \mathrm{H}, J_{2,3} 9.8 \mathrm{~Hz}, \mathrm{H}-2$ ), 5.70 (pseudo 
t, 2H, J $9.8 \mathrm{~Hz}, J 9.2 \mathrm{~Hz}$ in each, $\mathrm{H}-3, \mathrm{H}-4), 4.62\left(\mathrm{dd}, 1 \mathrm{H}, J_{6,6^{\prime}}, 12.5 \mathrm{~Hz}\right.$, $\left.J_{5,6} 2.6 \mathrm{~Hz}, \mathrm{H}-6\right), 4.46$ (dd, $\left.1 \mathrm{H}, J_{6,6^{\prime}} 12.6 \mathrm{~Hz}, J_{5,6^{\prime}} 5.9 \mathrm{~Hz}, \mathrm{H}_{\lambda}-6^{\prime}\right), 4.34$ (ddd, $\left.1 \mathrm{H}, J_{4,5} 9.8 \mathrm{~Hz}, J_{5,6} 5.9 \mathrm{~Hz}, J_{5,6^{\prime}} 2.6 \mathrm{~Hz}, \mathrm{H}-5\right), 1.33$ (s, 9H, $\left.\mathrm{C}_{4} \mathrm{H}_{9}\right) ;{ }^{13} \mathrm{C}$ NMR $\left(\mathrm{CDCl}_{3}, 90 \mathrm{MHz}\right): \delta(\mathrm{ppm}): 177.6\left(\mathrm{NHCOC}\left(\mathrm{CH}_{3}\right)_{3}\right)$, 166.0, 165.9, 164.9, 163.5 (CO), 133.5-127.6 (benzoyl $\operatorname{ArC}$ ), 114.4 $\left(\mathrm{CN},{ }^{3} \mathrm{~J}_{\mathrm{H}-2, \mathrm{CN}}=\sim 2.6 \mathrm{~Hz}\right), 77.8(\mathrm{C}-1), 71.6,70.6,69.6,68.5(\mathrm{C}-2-\mathrm{C}-$ 5), $67.0\left(\mathrm{NHCOC}\left(\mathrm{CH}_{3}\right)_{3}\right), 62.2(\mathrm{C}-6), 27.2\left(\mathrm{NHCOC}\left(\mathrm{CH}_{3}\right)_{3}\right)$ Anal. Calcd for $\mathrm{C}_{40} \mathrm{H}_{36} \mathrm{~N}_{2} \mathrm{O}_{10}$ (704.74): C, 68.17; H, 5.15; N, 3.97. Found: C, $68.00 ; \mathrm{H}, 4.86$; N, 4.11 .

3.2.11. $N$-Acetyl-2,3,4-tri-O-acetyl-1-cyano- $\beta$-D-arabinopyranosylamine (2-acetamido-3,4,5-tri-O-acetyl-2-deoxy- $\beta$-D-arabinohex-2-ulopyranosononitrile) (17)

Prepared from 7 ( $0.14 \mathrm{~g}, 0.36 \mathrm{mmol})$ in $\mathrm{CH}_{3} \mathrm{CN}$ according to Section 3.2. Reaction time $2 \mathrm{~d}$, purification by column chromatography $(1: 1 \rightarrow 3: 1$ EtOAc-Hexane), yield $0.05 \mathrm{~g}(41 \%)$ white crystals from $\mathrm{CH}_{2} \mathrm{Cl}_{2}-\mathrm{Et}_{2} \mathrm{O}$. Mp: $170-172{ }^{\circ} \mathrm{C} ;[\alpha]_{\mathrm{D}}-29$ (c 1.07, $\left.\mathrm{CHCl}_{3}\right) ;{ }^{1} \mathrm{H}$ NMR $\left(\mathrm{CDCl}_{3}, 360 \mathrm{MHz}\right): \delta(\mathrm{ppm}) 8.53(\mathrm{~s}, 1 \mathrm{H}, \mathrm{NH}), 5.69\left(\mathrm{~d}, 1 \mathrm{H}, J_{2,3}\right.$ $9.6 \mathrm{~Hz}, \mathrm{H}-2$ ), 5.34 (dd, $1 \mathrm{H}, J_{2,3} 9.6 \mathrm{~Hz}, J_{3,4} 5.0 \mathrm{~Hz}, \mathrm{H}-3$ ), 5.30 (ddd, $\left.1 \mathrm{H}, J_{3,4} 5.0 \mathrm{~Hz}, J_{4,5} 2.5 \mathrm{~Hz}, J_{4,5^{\prime}} 1.6 \mathrm{~Hz}, \mathrm{H}-4\right), 4.06$ (dd, $1 \mathrm{H}, J_{6,6^{\prime}}$ $\left.13.5 \mathrm{~Hz}, J_{5,6} 2.5 \mathrm{~Hz}, \mathrm{H}-5\right), 3.80$ (dd, $1 \mathrm{H}, J_{6,6^{\prime}} 13.5 \mathrm{~Hz}, J_{5,6} 1.6 \mathrm{~Hz}, \mathrm{H}-$ 5'), 2.13 (2), 2.08, 1.96 (3s, $\left.12 \mathrm{H}, \mathrm{OCOCH}_{3}, \mathrm{CH}_{3}\right) ;{ }^{13} \mathrm{C} \mathrm{NMR}\left(\mathrm{CDCl}_{3}\right.$, $90 \mathrm{MHz}): \delta(\mathrm{ppm}) 170.9\left(\mathrm{NHCOCH}_{3}\right), 170.1,169.8,168.2(\mathrm{CO})$, $114.7\left(\mathrm{CN},{ }^{3} J_{\mathrm{H}-2, \mathrm{CN}}=\sim 3.1 \mathrm{~Hz}\right), 78.0(\mathrm{C}-1), 67.9,66.9,66.8(\mathrm{C}-2-\mathrm{C}-$ 4), $61.6(\mathrm{C}-5), 23.0\left(\mathrm{CH}_{3}\right), 20.7,20.5,20.4\left(\mathrm{CH}_{3}\right)$; Anal. Calcd for $\mathrm{C}_{14} \mathrm{H}_{18} \mathrm{~N}_{2} \mathrm{O}_{8}$ (342.30): C, 49.12; H, 5.30; N, 8.18. Found: C, 50.10; H, 5.57; N, 8.33.

3.2.12. $N$-Propenoyl-2,3,4-tri-O-acetyl-1-cyano- $\beta$-D-arabinopyranosylamine (3,4,5-tri-O-acetyl-2-deoxy-2-propenamido- $\beta$-Darabino-hex-2-ulopyranosononitrile) (18)

Prepared from $7(0.31 \mathrm{~g}, 0.81 \mathrm{mmol})$ in $\mathrm{CH}_{2} \mathrm{CHCN}$ according to Section 3.2. Reaction time $2 \mathrm{~d}$, purification by column chromatography $(1: 1 \rightarrow 3: 1$ EtOAc-hexane), yield $0.12 \mathrm{~g}(43 \%)$ as a colourless syrup. $R_{\mathrm{f}}=0.28$ (1:1 EtOAc-hexane); $[\alpha]_{\mathrm{D}}-28\left(c 1.06, \mathrm{CHCl}_{3}\right) ;{ }^{1} \mathrm{H}$ NMR $\left(\mathrm{CDCl}_{3}, 200 \mathrm{MHz}\right): \delta(\mathrm{ppm}) 6.90(\mathrm{~s}, \hat{1} \mathrm{H}, \mathrm{NH}), 6.51(\mathrm{~d}, 1 \mathrm{H}, J$ $\left.7.5 \mathrm{~Hz}, \mathrm{CH}_{2}\right), 6.29(\mathrm{dd}, 1 \mathrm{H}, J 7.5 \mathrm{~Hz}, J 6.9 \mathrm{~Hz}, \mathrm{CH}), 5.86(\mathrm{~d}, 1 \mathrm{H}, J$ $7.5 \mathrm{~Hz}, \mathrm{CH}_{2}$ ), 5.77 (d, $\left.1 \mathrm{H}, J_{2,3} 8.8 \mathrm{~Hz}, \mathrm{H}-2\right), 5.28\left(\mathrm{dd}, 1 \mathrm{H}, J_{2,3} 8.8 \mathrm{~Hz}\right.$, $J_{3,4} 3.5 \mathrm{~Hz}, \mathrm{H}-3$ ), 5.24 (ddd, $1 \mathrm{H}, J_{3,4} 3.5 \mathrm{~Hz}, J_{4,5} 2.6 \mathrm{~Hz}, J_{4,5} 1.2 \mathrm{~Hz}$, $\mathrm{H}-4), 4.05-3.94$ ( $\left.\mathrm{m}, 2 \mathrm{H}, \mathrm{H}-5, \mathrm{H}-5^{\prime}\right), 2.18,2.17,2.06(3 \mathrm{~s}, 9 \mathrm{H}$, $\left.\mathrm{OCOCH}_{3}\right) ;{ }^{13} \mathrm{C} \quad \mathrm{NMR} \quad\left(\mathrm{CDCl}_{3}, \quad 50 \mathrm{MHz}\right): \delta(\mathrm{ppm}) \quad 170.0$ $\left(\mathrm{NHCOCH}=\mathrm{CH}_{2}\right), 169.6,168.3,165.6(\mathrm{CO}), 129.7\left(\mathrm{CH}_{2}=\mathrm{CH}\right), 128.9$ $\left(\mathrm{CH}_{2} \stackrel{\text { }}{=} \mathrm{CH}\right), 114.5\left(\mathrm{CN},{ }^{3} J_{\mathrm{H}-2, \mathrm{CN}}=\sim 3.1 \mathrm{~Hz}\right), 78.1(\mathrm{C}-1), 67.7,66.9$, 66.7 (C-2-C-4), 61.6 (C-5), $23.0\left(\mathrm{CH}_{3}\right), 20.7,20.5,20.4\left(\mathrm{CH}_{3}\right)$; Anal. Calcd for $\mathrm{C}_{15} \mathrm{H}_{18} \mathrm{~N}_{2} \mathrm{O}_{8}$ (354.31): C, 50.85; $\mathrm{H}, 5.12 ; \mathrm{N}, 7.91$. Found: $\mathrm{C}$, 50.30; H, 5.37; N, 8.13.

500 3.3. $\boldsymbol{N}$-(2,3,4,6-Tetra- $\boldsymbol{O}$-acetyl-1-bromo-1-deoxy- $\boldsymbol{\beta}$-D-galactopyranosylcarbonyl)]-L-alanine methylester $(\mathrm{N}$-(3,4,5,7-tetra-Oacetyl-2-bromo-2-deoxy- $\alpha$-D-galacto-hept-2-ulopyranosonoyl)L-alanine methylester) (20)

Pentachlorophenyl C-(2,3,4,6-tetra-O-acetyl-1-bromo-1-deoxy$\beta$-D-galactopyranosyl)formate ${ }^{32}(0.30 \mathrm{~g}, 0.43 \mathrm{mmol})$ was dissolved in dry 1,4-dioxane ( $3 \mathrm{~mL}$ ) and methyl L-alaninate. $\mathrm{HCl}$ (2 equiv), and $\mathrm{Et}_{3} \mathrm{~N}$ ( 2 equiv) were added. The reaction mixture was stirred at $\mathrm{rt}$ and monitored by TLC (1:1 EtOAc-hexane). After completion of the reaction the solvent was removed. The obtained crude product was purified by column chromatography (1:1 EtOAc-hexane) to give $0.18 \mathrm{~g}(73 \%) 20$ as colourless oil. $R_{\mathrm{f}}=0.24$ (1:1 EtOAc-hexane); $[\alpha]_{\mathrm{D}}+83\left(\right.$ c 0.73, $\left.\mathrm{CHCl}_{3}\right) ; \mathrm{IR} v_{\max }\left(\mathrm{CHCl}_{3}\right): 3380,2958,1758$, 1678, 1374, $1262,1070 \mathrm{~cm}^{-1}$; ${ }^{1} \mathrm{H} \mathrm{NMR}\left(\mathrm{CDCl}_{3}, 360 \mathrm{MHz}\right) \delta$ (ppm): $7.15(\mathrm{t}, 1 \mathrm{H}, J=6.8,6.8 \mathrm{~Hz}, \mathrm{NH}), 5.54$ (dd, $1 \mathrm{H}, J_{3,4} 3.2 \mathrm{~Hz}$, $\left.J_{4,5} 1.6 \mathrm{~Hz}, \mathrm{H}-4\right), 5.42$ (d, $1 \mathrm{H}, J_{2,3} 10.0 \mathrm{~Hz}, \mathrm{H}-2$ ), 5.32 (dd, $1 \mathrm{H}, J_{2,3}$ $\left.10.0 \mathrm{~Hz}, J_{3,4} 3.2 \mathrm{~Hz}, \mathrm{H}-3\right)$, 4.60-4.48 (m, 4H, H-5, H-6, H-6', CH), $3.79\left(\mathrm{~s}, 3 \mathrm{H}, \mathrm{OCH}_{3}\right), 2.18,2.12,2.09,1.98\left(4 \mathrm{~s}, 12 \mathrm{H}, \mathrm{OCOCH}_{3}\right), 1.45$ (d, 3H, J $\left.6.8 \mathrm{~Hz}, \mathrm{CH}\left(\mathrm{CH}_{3}\right)\right) ;{ }^{13} \mathrm{C} \mathrm{NMR}\left(\mathrm{CDCl}_{3}, 125 \mathrm{MHz}\right) \delta(\mathrm{ppm})$ : $172.5\left(\mathrm{COOCH}_{3}\right), 170.2,169.8,169.7,169.1(\mathrm{CO}), 164.2(\mathrm{CONH}$, $\left.{ }^{3} J_{\mathrm{H}-2, \mathrm{CONHCH} 2 \mathrm{CO} 2 \mathrm{Me}}=2.2 \mathrm{~Hz}\right), 94.3(\mathrm{C}-1), 73.3,69.7,66.4,66.4(\mathrm{C}-2-$ C-5), 60.5 (C-6), $52.5\left(\mathrm{COOCH}_{3}\right), 48.0(\mathrm{CH}), 20.8,20.7,20.5,20.4$ $\left(\mathrm{CH}_{3}\right)$, $17.6\left(\mathrm{CH}\left(\mathrm{CH}_{3}\right)\right.$. Anal. Calcd for $\mathrm{C}_{19} \mathrm{H}_{26} \mathrm{~N}_{1} \mathrm{Br}_{1} \mathrm{O}_{12}(540.32)$ : C, 42.24; H, 4.85; N, 2.59; Br, 14.79. Found: $C_{\lambda}, 41.80 ; \mathrm{H}, 4.68 ; \mathrm{N}$, $2.44 ; \mathrm{Br}, 15.10$.

\section{4. $N$-[(1R)-2,3,4,6-Tetra-O-acetyl-1,5-anhydro-D-galactitol-} spiro[1.4]-2-methyl-2-oxazolin-5-ylidene]glycine methylester (21)

Compound $\mathbf{1 9}^{32}(0.20 \mathrm{~g}, 0.38 \mathrm{mmol})$ was dissolved in dry $\mathrm{CH}_{3} \mathrm{CN}$ ( $2 \mathrm{~mL}$ ) and $\mathrm{Ag}_{2} \mathrm{CO}_{3}$ (1.1 equiv) was added. The solution was stirred at $\mathrm{rt}$ in the dark until complete transformation of the starting material (4 d, TLC 3:1 EtOAc-hexane). The mixture was filtrated on a Celite pad and the solvent was removed from the filtrate. The obtained crude product was purified by column chromatography $(3: 1$ EtOAc-hexane) to give $\mathbf{2 1}(0.09 \mathrm{~g}, 65 \%)$ as a colourless oil, $R_{\mathrm{f}}=0.39$ (3:1 EtOAc-hexane), $[\alpha]_{\mathrm{D}}+0.1\left(\mathrm{c} 0.44, \mathrm{CHCl}_{3}\right) ;{ }^{1} \mathrm{H}$ NMR $\left(\mathrm{CDCl}_{3}\right.$, $360 \mathrm{MHz}$ ): $\delta$ (ppm): 5.70 (d, $\left.1 \mathrm{H}, \widehat{J}_{2,3} 10.6 \mathrm{~Hz}, \mathrm{H}-2\right), 5.56$ (dd, $1 \mathrm{H}, J_{3,4}$ $\left.4.0 \mathrm{~Hz}, J_{4,5}<1 \mathrm{~Hz}, \mathrm{H}-4\right), 5.46$ (dd, $1 \mathrm{H}, J_{2,3} 10.6 \mathrm{~Hz}, J_{3,4} 4.0 \mathrm{~Hz}, \mathrm{H}-3$ ), 4.62 (ddd, $\left.1 \mathrm{H}, J_{5,6} 6.6 \mathrm{~Hz}, J_{5,6^{\prime}} 6.6 \mathrm{~Hz}, J_{4,5}<1 \mathrm{~Hz}, \mathrm{H}-5\right), 4.25$ (s, $2 \mathrm{H}$, $\mathrm{CH}_{2}$ ), 4.19 (dd, $\left.1 \mathrm{H}, J_{6,6^{\prime}} 11.9 \mathrm{~Hz}, J_{5,6} 6.6 \mathrm{~Hz}, \mathrm{H}-6\right), 4.10$ (dd, $1 \mathrm{H}, J_{6,6^{\prime}}$ $\left.11.9 \mathrm{~Hz}, J_{5,6^{\prime}} 6.6 \mathrm{~Hz}, \mathrm{H}-6^{\prime}\right), 3.77\left(\mathrm{~s}, 3 \mathrm{H}, \mathrm{OCH}_{3}\right), 2.25\left(\mathrm{~s}, 3 \mathrm{H}, \mathrm{CH}_{3}\right)$, $2.19,2.03,2.01,1.97\left(4 \mathrm{~s}, 12 \mathrm{H}, \mathrm{OCOCH}_{3}\right) ;{ }^{13} \mathrm{C} \mathrm{NMR}\left(\mathrm{CDCl}_{3}\right.$, $125 \mathrm{MHz}): \delta(\mathrm{ppm}): 170.4,170.2,170.0,168.7(\mathrm{CO}), 165.3(\mathrm{C}=\mathrm{N})$, $158.2\left(\mathrm{C}=\mathrm{N},{ }^{3} \mathrm{~J}_{\mathrm{H}-2, \mathrm{C}=\mathrm{NCH} 2 \mathrm{COOCH} 3}=\sim 3.1 \mathrm{~Hz}\right), 95.7(\mathrm{C}-1), 70.8, \widehat{6} 9.5$, 68.3, 67.9 (C-2-C-5), $61.4(\mathrm{C}-6), 52.1\left(\mathrm{OCH}_{3}\right), 50.3\left(\mathrm{CH}_{2}\right), 20.7,20.5$, 20.5, $20.4\left(\mathrm{OCOCH}_{3}\right), 14.9\left(\mathrm{CH}_{3}\right)$; Anal. Calcd for $\mathrm{C}_{20} \mathrm{H}_{26} \mathrm{~N}_{2} \mathrm{O}_{12}$ (486.44): C, 49.38; H, 5.39; N, 5.76. Found: C, 49.55; H, 5.56; N, 5.80.

\section{5. $N$-[(1R)-2,3,4,6-Tetra-O-acetyl-1,5-anhydro-D-galactitol- spiro[1.4]-2-benzyloxycarbonylaminomethyl-2-oxazolin-5- ylidene]glycine methylester (22)}

Compound $19(0.17 \mathrm{~g}, 0.33 \mathrm{mmol})$ was dissolved in dry $\mathrm{CH}_{3} \mathrm{NO}_{2}$ ( $3 \mathrm{~mL}$ ), $\mathrm{NCCH}_{2} \mathrm{NHCOOCH}_{2} \mathrm{Ph}$ (10 equiv) and $\mathrm{Ag}_{2} \mathrm{CO}_{3}$ (1.1 equiv) were added. The mixture was stirred in the dark at rt for $6 \mathrm{~d}$. After completion of the reaction (TLC, 3:1 EtOAc-hexane) the mixture was filtered on a Celite pad and the solvent was removed. The crude oil was purified by column chromatography (3:1 EtOAc-hexane) to give $0.04 \mathrm{~g}(29 \%)$ of 22 (1:1 mixture of two isomers) as a colourless oil. $R_{\mathrm{f}}=0.29$ (EtOAc); ${ }^{1} \mathrm{H}$ NMR $\left(\mathrm{CDCl}_{3}, 360 \mathrm{MHz}\right): \delta$ (ppm): 7.44-7.32 (m, 5H, Ph), $7.01(\mathrm{~s}, 1 \mathrm{H}, \mathrm{NH}), 5.71(\mathrm{~d}, 1 \mathrm{H}$, $\left.J_{2,3} 10.6 \mathrm{~Hz}, \mathrm{H}-2\right), 5.58-5.52\left(\mathrm{~m}, 2 \mathrm{H}, \mathrm{H}-4, \mathrm{H}-4^{\prime}\right), 5.48-5.30(\mathrm{~m}, 3 \mathrm{H}$, $\left.\mathrm{H}-2^{\prime}, \mathrm{H}-3, \mathrm{H}-3^{\prime}\right), \widehat{5} .20-5.14\left(\mathrm{~m}, 2 \mathrm{H}, \mathrm{CH}_{2}\right), \widehat{4} .60-4.62(\mathrm{~m}, 2 \hat{\mathrm{H}}, \mathrm{H}-5$, H-5'), 4.32-4.04 (m, 10H, H-6a, H-6a', H-6b, H-6b', $\mathrm{CH}_{2} \mathrm{a}, \mathrm{CH}_{2} \mathrm{~b}$, $\left.\mathrm{CH}_{2} \mathrm{Ph}\right), 3.79\left(\mathrm{~s}, 3 \mathrm{H}, \mathrm{OCH}_{3}\right), 2.19,2.17,2.13,2.11,2.03,2.01,1.99$, 1.97 (8s, $\left.24 \mathrm{H}, \mathrm{OCOCH}_{3}\right) ;{ }^{13} \mathrm{C} \mathrm{NMR}\left(\mathrm{CDCl}_{3}, 125 \mathrm{MHz}\right): \delta(\mathrm{ppm})$ : $170.5,170.3,170.1$ (2), 169.9, 169.4, 168.8 (2) (CO), 168.2 $\left(\mathrm{CO}_{2} \mathrm{CH}_{3}\right), 165.1(\mathrm{C}=\mathrm{N}), 157.1,157.0\left(\mathrm{CO}_{2} \mathrm{CH}_{2} \mathrm{Ph}\right), 135.9-128.2$ (aromatics), 95.3 (C-1), $94.1\left(\mathrm{C}-1^{\prime}\right), 73.6,71.0,69.8,69.5,68.3,67.9$, 67.5, $66.6\left(\mathrm{C}-2-\mathrm{C}-5\right.$ and $\left.\mathrm{C}^{-2} 2^{\prime}-\mathrm{C}-5^{\prime}\right), 71.0\left(\mathrm{CH}_{2}\right), 60.7(\mathrm{C}-6), 60.4$ $\left.\left(\mathrm{C}-6^{\prime}\right), 52.6\left(\mathrm{OCH}_{3}\right), 52.2 \hat{\mathrm{OCH}}_{3}{ }^{\prime}\right), 41.2(2)\left(\mathrm{CH}_{2}, \mathrm{CH}_{2}{ }^{\prime}\right), 20.9$ (6), $20.5\left(\mathrm{CH}_{3}\right)$

3.6. $N$-[(1R)-2,3,4,6-Tetra-O-acetyl-1,5-anhydro-D-galactitolspiro[1.4]-2-methyl-2-oxazolin-5-ylidene]-L-alanine methylester (23)

Compound 20 (0.60 g, $1.10 \mathrm{mmol})$ was dissolved in dry $\mathrm{CH}_{3} \mathrm{CN}$ $(10 \mathrm{~mL})$ and $\mathrm{Ag}_{2} \mathrm{CO}_{3}$ (1.1 equiv) was added. After stirring for $4 \mathrm{~d}$ in the dark the mixture wâs filtered on a Celite pad and the solvent was removed. The obtained crude product was purified by column chromatography (3:1 EtOAc-hexane) to give $0.24 \mathrm{~g}$ (43\%) 23 as a 
colourless oil. $R_{\mathrm{f}}=0.19\left(1: 1\right.$ EtOAc-hexane), $[\alpha]_{\mathrm{D}}+1$ (c 0.41, $\left.\mathrm{CHCl}_{3}\right)$; ${ }^{1} \mathrm{H} \mathrm{NMR}\left(\mathrm{CDCl}_{3}, 360 \mathrm{MHz}\right): \delta(\mathrm{ppm}): 5.65\left(\mathrm{~d}, 1 \mathrm{H}, J_{2,3} 10.6 \mathrm{~Hz}, \mathrm{H}-2\right)$, 5.54 (dd, $\left.1 \mathrm{H}, J_{3,4} 4.0 \mathrm{~Hz}, J_{4,5}<1 \mathrm{~Hz}, \mathrm{H}-4\right), 5.42$ (dd, $1 \mathrm{H}, J_{2,3} 10.6 \mathrm{~Hz}$, $J_{3,4} 4.0 \mathrm{~Hz}, \mathrm{H}-3$ ), 4.58 (ddd, $1 \mathrm{H}, J_{5,6} 6.6 \mathrm{~Hz}, J_{5,6^{\prime}} 6.6 \mathrm{~Hz}, J_{4,5}<1 \mathrm{~Hz}$, H-5), 4.42 (q, $1 \mathrm{H}, J 6.9 \mathrm{~Hz}, J 6.4 \mathrm{~Hz}, \mathrm{CH}), 4.18$ (dd, $1 \mathrm{H}, J_{6,6^{\prime}} 11.9 \mathrm{~Hz}$, $\left.J_{5,6} 6.6 \mathrm{~Hz}, \mathrm{H}-6\right), 4.11\left(1 \mathrm{H}, J_{6,6^{\prime}} 11.9 \mathrm{~Hz}, J_{5,6^{\prime}} 6.6 \mathrm{~Hz}, \mathrm{H}-6^{\prime}\right), 3.70$ (s, $\left.3 \mathrm{H}, \mathrm{OCH}_{3}\right), 2.24\left(\mathrm{~s}, 3 \mathrm{H}, \mathrm{CH}_{3}\right), 2.20,2.03,2.01,1.99(4 \mathrm{~s}, 12 \mathrm{H}$, $\left.\mathrm{OCOCH}_{3}\right), 1.48\left(\mathrm{~d}, 3 \mathrm{H}, \mathrm{J} 6.4 \mathrm{~Hz}, \mathrm{CH}\left(\mathrm{CH}_{3}\right)\right) ;{ }^{13} \mathrm{C} \mathrm{NMR}\left(\mathrm{CDCl}_{3}\right.$, $125 \mathrm{MHz}): \delta(\mathrm{ppm}): 171.3,170.4,170.2,169.9(\mathrm{CO}), 165.2(C=\mathrm{N})$, $155.8\left(C_{\bar{\lambda}}=\mathrm{N},{ }^{3} J_{\mathrm{H}-2, \mathrm{C}=\mathrm{NCH}-}=\sim 3.3 \mathrm{~Hz}\right), 95.5(\mathrm{C}-1), 70.6,69.5,68.4$, $67.9(\mathrm{C}-2-\mathrm{C}-5), 61.3(\mathrm{C}-6), 56.6(\mathrm{CH}), 52.0\left(\mathrm{OCH}_{3}\right), 20.6,20.5$, 20.4, $20.3\left(\mathrm{OCOCH}_{3}\right), 18.2\left(\mathrm{CH}\left(\mathrm{CH}_{3}\right), 14.9\left(\mathrm{CH}_{3}\right)\right.$; Anal. Calcd for $\mathrm{C}_{21} \mathrm{H}_{28} \mathrm{~N}_{2} \mathrm{O}_{12}$ (500.44): C, 51.12; H, 5.70; N, 6.26. Found: C, 50.85; H, 5.52; N, 5.96 .

\subsection{General procedure for the hydrolysis of spiro-oxazolines 21-23 to oligopeptides 24-26}

Compounds 21-23 each was dissolved in $\mathrm{CH}_{2} \mathrm{Cl}_{2}(1 \mathrm{~mL} /$ $0.1 \mathrm{mmol}), \mathrm{CF}_{3} \mathrm{COOH}$ or $\mathrm{AcOH}(1$ equiv) and water ( 2 equiv) were added. The mixture was stirred at rt until disappearance of the starting material (TLC, 3:1 EtOAc-hexane). It was then diluted with $\mathrm{CH}_{2} \mathrm{Cl}_{2}$ and washed with satd aq $\mathrm{NaHCO}_{3}$ solution $(2 \times 5 \mathrm{~mL})$, water $(5 \mathrm{~mL})$, dried, and the solvent removed. The obtained syrup was purified by column chromatography.

3.7.1. $N$-(2,3,4,6-Tetra-O-acetyl-1-acetamido-1-deoxy- $\beta$-D-galactopyranosylcarbonyl)glycine methylester ( $N$-(2-acetamido-3,4,5,7-tetra- 0 -acetyl-2-deoxy- $\alpha$-D-galacto-hept-2-ulopyranosonoyl)glycine methylester) (24)

Prepared from 21 (0.05 g, $0.10 \mathrm{mmol})$ according to General procedure 3.7. Purified by column chromatography (9:1 EtOAc$\mathrm{MeOH})$, yield $(0.02 \mathrm{~g}, 55 \%)$ of $\mathbf{2 4}$ as a colourless syrup. $R_{\mathrm{f}}=0.42$ (9:1 EtOAc-hexane); $[\alpha]_{\mathrm{D}}+69\left(\mathrm{c} 0.38, \mathrm{CHCl}_{3}\right) ;{ }^{1} \mathrm{H}$ NMR $\left(\mathrm{CDCl}_{3}\right.$, $360 \mathrm{MHz}): \delta(\mathrm{ppm}): 7.28(\mathrm{t}, 1 \hat{\mathrm{H}}, J 5.8 \mathrm{~Hz}, J 5.8 \mathrm{~Hz}, \mathrm{NH}), 6.37$ (s, $1 \mathrm{H}, \mathrm{NH}), 5.45\left(\mathrm{~d}, 1 \mathrm{H}, J_{2,3} 10.6 \mathrm{~Hz}, \mathrm{H}-2\right), 5.56\left(\mathrm{dd}, 1 \mathrm{H}, J_{3,4} 3.3 \mathrm{~Hz}\right.$, $\left.J_{4,5} 1.1 \mathrm{~Hz}, \mathrm{H}-4\right), 5.22$ (dd, $\left.1 \mathrm{H}, J_{2,3} 10.6 \mathrm{~Hz}, J_{3,4} 3.3 \mathrm{~Hz}, \mathrm{H}-3\right), 4.33-$ $3.98\left(\mathrm{~m}, 5 \mathrm{H}, \mathrm{H}-5, \mathrm{H}-6, \mathrm{H}^{\prime} \mathrm{6}^{\prime}, \mathrm{CH}_{2}\right), 3.78\left(\mathrm{~s}, 3 \mathrm{H}, \mathrm{OCH}_{3}\right), 2.20,2.09$, $2.06,1.99\left(4 \mathrm{~s}, 12 \mathrm{H}, \mathrm{OCOCH}_{3}\right), 2.09\left(\mathrm{~s}, 3 \mathrm{H}, \mathrm{NHCOCH}_{3}\right) ;{ }^{13} \mathrm{C} \mathrm{NMR}$ $\left(\mathrm{CDCl}_{3}, 125 \mathrm{MHz}\right): \delta$ (ppm): 170.9, 170.5, 170.1, 169.5 (CO), 170.1 $\left(\mathrm{CO}_{2} \mathrm{CH}_{3}\right), \quad 168.6 \quad\left(\mathrm{NHCOCH}_{3}\right), 167.3 \quad\left(\mathrm{CONHCH}_{2}, \quad{ }^{3} J_{\mathrm{H}-2, \mathrm{CON}-}\right.$ $\mathrm{HCH} 2 \mathrm{COOCH} 3=\sim 2.4 \mathrm{~Hz}), 83.7(\mathrm{C}-1), 68.7,68.4,67.0$ (2) (C-2-C-5), 60.8 (C-6), $52.3\left(\mathrm{OCH}_{3}\right), 41.3\left(\mathrm{CH}_{2}\right), 23.5\left(\mathrm{NHCOCH}_{3}\right), 20.6(3)$, $20.5\left(\mathrm{CH}_{3}\right)$; Anal. Calcd for $\mathrm{C}_{20} \mathrm{H}_{28} \mathrm{~N}_{2} \mathrm{O}_{13}$ (504.45): $\mathrm{C}, 47.62 ; \mathrm{H}$, 5.59; N, 5.55. Found: C, 47.44; H, 5.36; N, 5.65.

$620 \quad 3.7 .2$. $N$-(2,3,4,6-Tetra-O-acetyl-1-Z-glycylamido-1-deoxy- $\boldsymbol{\beta}$-Dgalactopyranosylcarbonyl)glycine methylester $(N-(3,4,5,7-$ tetra-O-acetyl-2-deoxy-2-Z-glycylamido- $\alpha$-D-galacto-hept2-ulopyranosonoyl)glycine methylester) (25)

Prepared from $22(0.04 \mathrm{~g}, 0.06 \mathrm{mmol})$ according to Section 3.7. Purified by column chromatography (9:1 EtOAc-MêOH), yield $0.01 \mathrm{~g}(27 \%)$ of $\mathbf{2 4}$ as a colourless oil. $R_{\mathrm{f}}=0.13$ (5:1 EtOAc-hexane); $[\alpha]_{\mathrm{D}}+58\left(c \quad 0.160, \mathrm{CHCl}_{3}\right) ;{ }^{1} \mathrm{H}$ NMR $\left(\mathrm{CDCl}_{3}, 360 \mathrm{MHz}\right): \delta(\mathrm{ppm})$ : 7.38-7.32(m, 5H, ArH), $7.30(\mathrm{~s}, 1 \mathrm{H}, \mathrm{NH}), 7.18(\mathrm{~s}, 1 \mathrm{H}, \mathrm{NH}), 5.51(\mathrm{~s}$, $1 \mathrm{H}, \mathrm{NH}), 5.46$ (d, $\left.1 \mathrm{H}, J_{2,3} 10.6 \mathrm{~Hz}, \mathrm{H}-2\right), 5.44\left(\mathrm{dd}, 1 \mathrm{H}, J_{3,4} 3.4 \mathrm{~Hz}\right.$, $\left.J_{4,5} 1.1 \mathrm{~Hz}, \mathrm{H}-4\right), 5.19$ (dd, $1 \mathrm{H}, J_{2,3} 10.6 \mathrm{~Hz}, J_{3,4} 3.4 \mathrm{~Hz}, \mathrm{H}-3$ ), $5.12-$ $5.08\left(\mathrm{~m}, 2 \mathrm{H}, \mathrm{CH}_{2}\right), 4.29-3.86\left(\mathrm{~m}, 5 \mathrm{H}, \mathrm{H}-5, \mathrm{H}-6, \mathrm{H}-6^{\prime}, \mathrm{CH}_{2}\right), 3.77(\mathrm{~s}$, $\left.3 \mathrm{H}, \mathrm{OCH}_{3}\right), 2.20, \hat{2} .06,2.05,1.95\left(4 \mathrm{~s}, 12 \mathrm{H}, \mathrm{OCOCH}_{3}\right) ;{ }^{13} \mathrm{C} \mathrm{NMR}$ $\left(\mathrm{CDCl}_{3}, 125 \mathrm{MHz}\right): \delta(\mathrm{ppm}): 170.6,170.2,170,169.9$ (2), 169.5, 168.8, $168.7\left(\mathrm{CO}_{2} \mathrm{CH}_{3}, \mathrm{NHCOCH}_{2}, \mathrm{NHCO}_{2}, \mathrm{CO}\right), 166.7\left(\mathrm{CONCH}_{2}\right.$, $\left.{ }^{3} J_{\mathrm{H}-2, \mathrm{CONHCH} 2 \mathrm{COOCH} 3}=\sim 2.9 \mathrm{~Hz}\right) ; 156.8\left(-\mathrm{CO}_{2} \mathrm{CH}_{2} \mathrm{Ph}\right), 133.0-128.6$ (aromatics), 83.8 (C-1), 69.2, 68.5, 67.1 (2) (C-2-C-5), $67.1\left(\mathrm{CH}_{2}\right)$, $65.6\left(\mathrm{CH}_{2}\right), 60.8(\mathrm{C}-6), 52.4\left(\mathrm{OCH}_{3}\right), 41.4(2)\left(\mathrm{CH}_{2}\right), 20.6(6), 20.5$ (2) $\left(\mathrm{CH}_{3}\right)$; Anal. Calcd for $\mathrm{C}_{28} \mathrm{H}_{35} \mathrm{~N}_{3} \mathrm{O}_{15}$ (653.60): $\mathrm{C}, 51.46 ; \mathrm{H}, 5.40$; N, 6.43. Found: C, 51.65; H, 5.56; N, 6.72.
3.7.3. $N$-(2,3,4,6-Tetra-O-acetyl-1-acetamido-1-deoxy- $\beta$-D-galactopyranosylcarbonyl)-L-alanine methylester ( $\mathrm{N}$-(2-acetamido3,4,5,7-tetra-O-acetyl-2-deoxy- $\alpha$-D-galacto-hept-2-ulopyranosonoyl)-L-alanine methylester) (26)

Prepared from $23(0.05 \mathrm{~g}, 0.10 \mathrm{mmol})$ according to Section 3.7 . Purified by column chromatography (3:1 EtOAc-hexane), yield $0.015 \mathrm{~g}(29 \%)$ of $\mathbf{2 6}$ as colourless oil. $R_{\mathrm{f}}=0.15$ (EtOAc); $[\alpha]_{\mathrm{D}}+69.0$ $\left(c 0.40, \mathrm{CHCl}_{3}\right) ;{ }^{1} \mathrm{H} \mathrm{NMR}\left(\mathrm{CDCl}_{3}, 360 \mathrm{MHz}\right): \delta(\mathrm{ppm}): 7.28(\mathrm{t}, 1 \mathrm{H}, J$ $5.8 \mathrm{~Hz}, J 5.8 \mathrm{~Hz}, \mathrm{NH}), 6.27(\mathrm{~s}, 1 \mathrm{H}, \mathrm{NH}), 5.46-5.42(\mathrm{~m}, 2 \mathrm{H}, \mathrm{H}-2, \mathrm{H}-$ 4), 5.19 (dd, $1 \mathrm{H}, J_{2,3} 10.6 \mathrm{~Hz}, J_{3,4} 3.3 \mathrm{~Hz}, \stackrel{\mathrm{H}}{-}-3$ ), 4.52 (q, $1 \mathrm{H}, J 6.8 \mathrm{~Hz}$, J $6.4 \mathrm{~Hz}, \mathrm{CH}), 4.35\left(\mathrm{dd}, 1 \mathrm{H}, J_{\xi_{2}, 6^{\prime}} 11.9 \mathrm{~Hz}, J_{5,6} 6.6 \mathrm{~Hz}, \mathrm{H}-6\right), 4.25$ (ddd, $\left.1 \mathrm{H}, J_{5,6} 6.6 \mathrm{~Hz}, J_{5,6^{\prime}} 6.6 \mathrm{~Hz}, J_{4,5} 0.9 \mathrm{~Hz}, \mathrm{H}-5\right), 4.14\left(1 \mathrm{H}, \mathrm{dd}, J_{6,6^{\prime}}\right.$ $\left.11.9 \mathrm{~Hz}, J_{5,6^{\prime}} 6.6 \mathrm{~Hz}, \mathrm{H}-6^{\prime}\right), 3.79\left(\mathrm{~s}, 3 \mathrm{H}, \mathrm{OCH}_{3}\right), 2.23(\mathrm{~s}, 3 \mathrm{H}, \mathrm{NHC}-$ $\left.\mathrm{OCH}_{3}\right) ; 2.10,2.07,2.06,2.00\left(4 \mathrm{~s}, 12 \mathrm{H}, \mathrm{OCOCH}_{3}\right), 1.46(\mathrm{~d}, 3 \mathrm{H}, J$ $\left.6.4 \mathrm{~Hz}, \mathrm{CH}\left(\mathrm{CH}_{3}\right)\right) ;{ }^{13} \mathrm{C}$ NMR $\left(\mathrm{CDCl}_{3}, 125 \mathrm{MHz}\right): \delta$ (ppm): 173.1, 171.3, 171.0, 170.7 (CO), $170.5\left(\mathrm{CO}_{2} \mathrm{CH}_{3}\right), 168.9\left(\mathrm{NHCOCH}_{3}\right), 166.7$ $\left(\mathrm{CONHCH}_{2},{ }^{3} \mathrm{~J}_{\mathrm{H}-2, \mathrm{CONHCH} 2 \mathrm{COOCH} 3}=\sim 2.4 \mathrm{~Hz}\right), 83.9(\mathrm{C}-1), 69.2,68.9$, 67.4 (2) (C-2-C-5), 60.8 (C-6), $52.8\left(\mathrm{OCH}_{3}\right), 48.9(\mathrm{CH}), 24.0$ $\left(\mathrm{NHCOCH}_{3}\right), 20.9,20.6,(2), 20.5\left(\mathrm{CH}_{3}\right), 18.4\left(\mathrm{CH}\left(\mathrm{CH}_{3}\right)\right)$; Anal. Calcd for $\mathrm{C}_{21} \mathrm{H}_{30} \mathrm{~N}_{2} \mathrm{O}_{13}$ (518.45): C, 49.90; $\mathrm{H}, 5.72 ; \mathrm{N}, 5.60$. Found: $\mathrm{C}_{\Lambda}$ 49.63; H, 5.56; N, 5.44 .

3.8. $N$-(2,3,4,6-Tetra-O-acetyl-1-amino-1-deoxy- $\alpha$-D-galactopyranosylcarbonyl)glycine methylester $(N-(3,4,5,7-$ tetra- $O$-acetyl2-amino-2-deoxy- $\beta$-D-galacto-hept-2-ulopyranosonoyl)glycine methylester) (28)

Azide $27^{32}(0.10 \mathrm{~g}, 0.20 \mathrm{mmol})$ was dissolved in dry EtOAc $(5 \mathrm{~mL})$ and Raney-Ni ( $\sim 0.2 \mathrm{~g}, 2$ equiv) was added to the solution. The mixture was stirred at $7 \hat{0}^{\circ} \mathrm{C}$ and monitored by TLC $(3: 1$ EtOAc-hexane). After completion of the reaction the mixture was filtered on a Celite pad and the solvent was removed. The obtained syrup was crystallised from EtOH to give $0.07 \mathrm{~g}$ (79\%) of $\mathbf{2 8}$ as a white crystalline product. Mp: $123-126^{\circ} \mathrm{C} ;[\alpha]_{\mathrm{D}}+77(\mathrm{c} 1.06$, $\left.\mathrm{CHCl}_{3}\right) ;{ }^{1} \mathrm{H} \mathrm{NMR}\left(\mathrm{CDCl}_{3}, 360 \mathrm{MHz}\right): \delta(\mathrm{ppm}): 7.53(\mathrm{t}, 1 \mathrm{H}, \hat{J} 5.3 \mathrm{~Hz}$, J $5.3 \mathrm{~Hz}, \mathrm{NH}), 5.62$ (dd, $\left.1 \mathrm{H}, J_{2,3} 11.0 \mathrm{~Hz}, J_{3,4} 3.2 \mathrm{~Hz}, \mathrm{H}-3\right), 5.50$ (dd, $\left.1 \mathrm{H}, J_{3,4} 3.2 \mathrm{~Hz}, J_{4,5}<1 \mathrm{~Hz}, \mathrm{H}-4\right), 5.24$ (d, $\left.1 \mathrm{H}, J_{2,3} 11.0 \mathrm{~Hz}, \mathrm{H}-2\right), 5.13$ (ddd, $1 \mathrm{H}, J_{5,6} 6.3 \mathrm{~Hz}, J_{5,6^{\prime}} 6.3, J_{4,5}<1 \mathrm{~Hz}, \mathrm{H}-5$ ), 4.18 (dd, $1 \mathrm{H}, J$ $18.4 \mathrm{~Hz}, J 5.3 \mathrm{~Hz}, \mathrm{CH}_{2}$ ), 4.10 (dd, $1 \mathrm{H}, J_{6,6^{\prime}} 11.5 \mathrm{~Hz}, J_{5,6} 6.3 \mathrm{~Hz}, \mathrm{H}-6$ ), 4.02 (dd, $\left.1 \mathrm{H}, J_{6,6^{\prime}} 11.0 \mathrm{~Hz}, J_{5,6^{\prime}} 6.3 \widehat{\mathrm{Hz}}, \mathrm{H}-6^{\prime}\right), 3.95$ (dd, $1 \mathrm{H}, J$ $\left.18.4 \mathrm{~Hz}, J 5.3 \mathrm{~Hz}, \mathrm{CH}_{2}\right), 3.78\left(\mathrm{~s}, 3 \mathrm{H}, \mathrm{OCH}_{3}\right) ; \hat{2} .29\left(\mathrm{~s}, 2 \mathrm{H}, \mathrm{NH}_{2}\right), 2.16$,

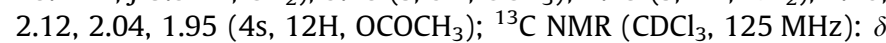
(ppm): $170.4\left(\mathrm{CO}_{2} \mathrm{CH}_{3}\right), 170.3,170.1,169.8,169.6(\mathrm{CO}), 169.5$ $\left(\mathrm{CONH},{ }^{3} \mathrm{~J}_{\mathrm{H}-2, \mathrm{CONHCH} 2 \mathrm{COOCH} 3}=\sim 5.8 \mathrm{~Hz}\right), 86.5(\mathrm{C}-1), 71.3,70.4,68.9$, 68.0 (C-2-C-5), 62.0 (C-6), $52.3\left(\mathrm{COOCH}_{3}\right), 40.7\left(\mathrm{CH}_{2}\right), 20.6(2)$, 20.5 (2) (CO); Anal. Calcd for $\mathrm{C}_{18} \mathrm{H}_{26} \mathrm{~N}_{2} \mathrm{O}_{12}$ (462.41): C, 46.75; $\mathrm{H}$, 5.67; N, 6.06. Found: C, 46.45; H, 5.76; N, 5.9े0.

3.9. $N$-(2,3,4,6-Tetra-O-acetyl-1-acetamido-1-deoxy- $\alpha$-D-galactopyranosylcarbonyl)glycine methylester $(N-(3,4,5,7$-tetra-Oacetyl-2-acetamido-2-deoxy- $\beta$-D-galacto-hept-2-ulopyranosonoyl)glycine methylester) (29)

Compound 28 ( $0.26 \mathrm{~g}, 0.56 \mathrm{mmol})$ was dissolved in dry pyridine $(5 \mathrm{~mL})$ and $\mathrm{AcCl}(0.08 \mathrm{~mL}, 1.12 \mathrm{mmol})$ was added. The mixture was stirred at rt overnight and monitored by TLC (99:1 EtOAc-MeOH). The volatiles were then removed, followed by co-evaporations with $\mathrm{PhCH}_{3}(2 \times 5 \mathrm{~mL})$. The residue was dissolved in EtOAc $(10 \mathrm{~mL})$ and washed with water. The water phase was washed with EtOAc $(5 \times 10 \mathrm{~mL})$. The combined organic phase was dried, the solvent removed, and the residue purified by column chromatography (99:1 EtOAc-MeOH) to give $0.045 \mathrm{~g}$, (15\%) of compound 29 as a colourless oil in the first fraction $\left(R_{\mathrm{f}}=0.23,99: 1\right.$ EtOAc-MeOH $)$ and $0.045 \mathrm{~g}(15 \%)$ of compound 24 as the second fraction $\left(R_{\mathrm{f}}=0.31,99: 1\right.$ EtOAc-MeOH $)$. 
Characterisation data for 29: $[\alpha]_{\mathrm{D}}+153\left(\mathrm{c} 0.078, \mathrm{CHCl}_{3}\right) ;{ }^{1} \mathrm{H} \mathrm{NMR}$ $\left(\mathrm{CDCl}_{3}, 360 \mathrm{MHz}\right): \delta(\mathrm{ppm}): 7.86(\mathrm{t}, 1 \mathrm{H}, J 5.8 \mathrm{~Hz}, J 5.8 \mathrm{~Hz}, \mathrm{NH}), 7.06$ (s, $1 \mathrm{H}, \mathrm{NH}), 5.63$ (dd, $1 \mathrm{H}, J_{2,3} 10.5 \mathrm{~Hz}, J_{3,4} 3.3 \mathrm{~Hz}, \mathrm{H}-3$ ), 5.47 (dd, $1 \mathrm{H}$, $\left.J_{3,4} 3.3 \mathrm{~Hz}, J_{4,5} 1.0 \mathrm{~Hz}, \mathrm{H}-4\right), 5.23$ (d, $\left.1 \mathrm{H}, J_{2,3} 10.5 \mathrm{~Hz}, \mathrm{H}-2\right), 4.60$ (pseudo t, 1H, $\left.J_{5,6} 6.7 \mathrm{~Hz}, J_{5,6^{\prime}} 6.4 \mathrm{~Hz}, \mathrm{H}-5\right), 4.14-3.05$ (m, 4H, H-6, $\left.\mathrm{H}-6^{\prime}, \mathrm{CH}_{2}\right), 3.74\left(\mathrm{~s}, 3 \mathrm{H}, \mathrm{OCH}_{3}\right), 2.15,2.14,2.02,1.96(4 \mathrm{~s}, 12 \mathrm{H}$, $\left.\mathrm{OCOCH}_{3}\right), 2.01\left(\mathrm{~s}, 3 \mathrm{H}, \mathrm{NHCOCH}_{3}\right) ;{ }^{13} \mathrm{C} \mathrm{NMR}\left(\mathrm{CDCl}_{3}, 125 \mathrm{MHz}\right): \delta$ (ppm): 172.7, 170.2 (3), $169.6\left(\mathrm{CO}, \mathrm{COOCH}_{3}\right), 169.3\left(\mathrm{NHCOCH}_{3}\right)$, $166.3\left(\mathrm{CONHCH}_{2},{ }^{3} J_{\mathrm{H}-2, \mathrm{CONHCH} 2 \mathrm{COOCH}_{3}}=\sim 5.3 \mathrm{~Hz}\right), 85.8(\mathrm{C}-1), 71.1$, 70.1, 68.1, 67.2 (C-2-C-5), $61.2(\mathrm{C}-6), 52.4\left(\mathrm{OCH}_{3}\right), 41.1\left(\mathrm{CH}_{2}\right)$, $24.1\left(\mathrm{NHCOCH}_{3}\right), 20.9,20.620 .5$ (2) $\left(\mathrm{CH}_{3}\right)$; Anal. Calcd for $\mathrm{C}_{20} \mathrm{H}_{28} \mathrm{~N}_{2} \mathrm{O}_{13}$ (504.45): C, 47.62; H, 5.59; N, 5.55. Found: C, 47.14; $\mathrm{H}, 5.31 ; \mathrm{N}, 5.25$.

\section{Acknowledgements}

This work was supported by the Hungarian Scientific Research Fund (Grant OTKA NK 68578) as well as by the TÁMOP 4.2.1/B09/1/KONV-2010007 project co-financed by the European Union and the European Social Fund.

\section{References}

1. Bishop, R. In Comprehensive Organic Synthesis; Trost, B. M., Fleming, I., Winterfeldt, E., Eds.; Pergamon Press: Oxford, 1991; pp 261-300.

2. Kürti, L.; Czakó, B. Strategic Applications of Named Reactions in Organic Synthesis: Elsevier, 2005. pp 382-383.

3. Fügedi, P. In The Organic Chemistry of Sugars; Levy, D. E., Fügedi, P., Eds.; CRC Press: Boca Raton, 2006; pp 89-179.

4. Braccini, I.; Derouet, C.; Esnault, J.; Dupenhoat, C. H.; Mallet, J. M.; Michon, V.; Sinaÿ, P. Carbohydr. Res. 1993, 246, 23-41.

5. Ratcliffe, A. J.; Fraser-Reid, B. J. Chem. Soc., Perkin Trans. 1 1989, 1805-1810.

6. Pougny, J. R.; Sinaÿ, P. Tetrahedron Lett. 1976, 4073-4076.

7. Ratcliffe, A. J.; Fraser-Reid, B. J. Chem. Soc., Perkin Trans. 1 1990, 747-750.

8. Rao, C. S.; Ratcliffe, A. J.; Fraser-Reid, B. J. Chem. Soc., Perkin Trans. 1 1993, $1207-$ 1211.

9. Ratcliffe, A. J.; Konradsson, P.; Fraserreid, B. J. Am. Chem. Soc. 1990, 112, 5665 5667.

10. Ratcliffe, A. J.; Konradsson, P.; Fraser-Reid, B. Carbohydr. Res. 1991, 216, 323 335.

11. Nair, L. G.; Fraser-Reid, B.; Szardenings, A. K. Org. Lett. 2001, 3, 317-319.

12. Schweizer, F.; Lohse, A.; Otter, A.; Hindsgaul, O. Synlett 2001, 1434-1436.

13. Lohse, A.; Schweizer, F.; Hindsgaul, O. Comb. Chem. High Throughput Screening 2002, 5, 389-394.

14. Penner, M.; Taylor, D.; Desautels, D.; Marat, K.; Schweizer, F. Synlett 2005, 212216.

15. Penner, M.; Schweizer, F. Carbohydr. Res. 2007, 342, 7-15.
16. Gordon, D. M.; Danishefsky, S. J. J. Org. Chem. 1991, 56, 3713-3715.

17. Pavia, A. A.; Ungchhun, S. N.; Durand, J. L. J. Org. Chem. 1981, 46, 3158-3160.

18. Noort, D.; Vandermarel, G. A.; Mulder, G. J.; Vanboom, J. H. Synlett 1992, 224226.

19. Blanco, J. L. J.; Rubio, E. M.; Mellet, C. O.; Fernandez, J. M. G. Synlett 2004, 22302232.

20. Marra, A.; Sinaÿ, P. Carbohydr. Res. 1990, 200, 319-337.

21. Heinemann, F.; Hiegemann, M.; Welzel, P. Tetrahedron 1992, 48, 3781-3788.

22. Handlon, A. L.; Fraserreid, B. J. Am. Chem. Soc. 1993, 115, 3796-3797.

23. Elias, C.; Gelpi, M. E.; Cadenas, R. A. J. Carbohydr. Chem. 1995, 14, 1209-1216.

24. Song, X. Z.; Hollingsworth, R. I. Synlett 2006, 3451-3454.

25. Klemer, A.; Kohla, M. J. Carbohydr. Chem. 1988, 7, 785-797.

26. Wang, Z. D.; Sheikh, S. O.; Cox, S.; Zhang, Y. L.; Massey, K. Eur. J. Org. Chem. 2007, 2243-2247.

27. Gyóllai, V.; Somsák, L.; Szilágyi, L. Tetrahedron Lett. 1999, 40, 3969-3972.

28. Kiss, L.; Somsák, L. Carbohydr. Res. 1996, 291, 43-52.

29. Shimizu, M.; Togo, H.; Yokoyama, M. Synthesis 1998, 799-822.

30. Yokoyama, M. Carbohydr. Res. 2000, 327, 5-14.

31. Gyóllai, V.; Somsák, L.; Györgydeák, Z. Tetrahedron 1998, 54, 13267-13276.

32. Czifrák, K.; Szilágyi, P.; Somsák, L. Tetrahedron: Asymmetry 2005, 16, 127-141.

33. Czifrák, K.; Kovács, L.; Kövér, K. E.; Somsák, L. Carbohydr. Res. 2005, 340, 2328 2334.

34. Májer, G.; Borbás, A.; Illyés, T. Z.; Szilágyi, L.; Bényei, A. C.; Lipták, A. Carbohydr. Res. 2007, 342, 1393-1404.

35. Ősz, E.; Sós, E.; Somsák, L.; Szilágyi, L.; Dinya, Z. Tetrahedron 1997, 53, 58135824.

36. Somsák, L.; Kovács, L.; Gyóllai, V.; Ösz, E. Chem. Commun. 1999, 591-592.

37. Somsák, L.; Kovács, L.; Tóth, M.; Ősz, E.; Szilágyi, L.; Györgydeák, Z.; Dinya, Z.; Docsa, T.; Tóth, B.; Gergely, P. J. Med. Chem. 2001, 44, 2843-2848.

38. Somsák, L.; Nagy, V. Tetrahedron: Asymmetry 2000, 11, 1719-1727. Corrigendum 2247.

39. Williamson, R. T.; Marquez, B. L.; Gerwick, W. H.; Kövér, K. E. Magn. Reson. Chem. 2000, 38, 265-273.

40. Dondoni, A.; Marra, A. Chem. Rev. 2000, 100, 4395-4421.

41. Gruner, S. A. W.; Locardi, E.; Lohof, E.; Kessler, H. Chem. Rev. 2002, 102, 491514.

42. Schweizer, F. Angew. Chem., Int. Ed. 2002, 41, 230-253.

43. Risseeuw, M. D. P.; Overhand, M.; Fleet, G. W. J.; Simone, M. I. Tetrahedron: Asymmetry 2007, 18, 2001-2010.

44. Yamanoi, T.; Matsuda, S. Heterocycles 2009, 79, 163-194.

45. Estevez, J. C.; Estevez, R. J.; Ardron, H.; Wormald, M. R.; Brown, D.; Fleet, G. W. J. Tetrahedron Lett. 1994, 35, 8885-8888.

46. Estevez, J. C.; Ardron, H.; Wormald, M. R.; Brown, D.; Fleet, G. W. J. Tetrahedron Lett. 1994, 35, 8889-8890.

47. Estevez, J. C.; Long, D. D.; Wormald, M. R.; Dwek, R. A.; Fleet, G. W. J. Tetrahedron Lett. 1995, 36, 8287-8290.

48. Estevez, J. C.; Smith, M. D.; Lane, A. L.; Crook, S.; Watkin, D. J.; Besra, G. S.; Brennan, P. J.; Nash, R. J.; Fleet, G. W. J. Tetrahedron: Asymmetry 1996, 7, 387390.

49. Long, D. D.; Tennant-Eyles, R. J.; Estevez, J. C.; Wormald, M. R.; Dwek, R. A.; Smith, M. D.; Fleet, G. W. J. J. Chem. Soc., Perkin Trans. 1 2001, 807-813.

50. Bleriot, Y.; Simone, M. I.; Wormald, M. R.; Dwek, R. A.; Watkin, D. J.; Fleet, G. W. J. Tetrahedron: Asymmetry 2006, 17, 2276-2286. 